Article

\title{
Nutritional Enhancement of Health Beneficial Omega-3 \\ Long-Chain Polyunsaturated Fatty Acids in the Muscle, Liver, Kidney, and Heart of Tattykeel Australian White MARGRA Lambs Fed Pellets Fortified with Omega-3 Oil in a Feedlot System
}

\author{
Shedrach Benjamin Pewan ${ }^{1,2} \mathbb{D}^{\mathbb{D}}$, John Roger Otto ${ }^{1}\left(\mathbb{D}\right.$, Robert Tumwesigye Kinobe $^{1}(\mathbb{D}$, \\ Oyelola Abdulwasiu Adegboye ${ }^{3}$ (D) and Aduli Enoch Othniel Malau-Aduli 1,*(D)
}

1 Animal Genetics and Nutrition, Veterinary Sciences Discipline, College of Public Health, Medical and Veterinary Sciences, Division of Tropical Health and Medicine, James Cook University, Townsville, QLD 4811, Australia; shedrach.pewan@my.jcu.edu.au (S.B.P.); john.otto@jcu.edu.au (J.R.O.); robert.kinobe@jcu.edu.au (R.T.K.)

2 National Veterinary Research Institute, Private Mail Bag 01 Vom, Plateau State, Nigeria

check for updates

Citation: Pewan, S.B.; Otto, J.R.; Kinobe, R.T.; Adegboye, O.A.; Malau-Aduli, A.E.O. Nutritional Enhancement of Health Beneficial Omega-3 Long-Chain

Polyunsaturated Fatty Acids in the Muscle, Liver, Kidney, and Heart of Tattykeel Australian White MARGRA Lambs Fed Pellets Fortified with Omega-3 Oil in a Feedlot System. Biology 2021, 10, 912. https:// doi.org/10.3390/biology10090912

Academic Editors: Zipei Zhang, Quancai Sun and Xian Wu

Received: 31 August 2021 Accepted: 10 September 2021 Published: 14 September 2021

Publisher's Note: MDPI stays neutral with regard to jurisdictional claims in published maps and institutional affiliations.

Copyright: (C) 2021 by the authors Licensee MDPI, Basel, Switzerland. This article is an open access article distributed under the terms and conditions of the Creative Commons Attribution (CC BY) license (https:// creativecommons.org/licenses/by/ $4.0 /)$.
3 Public Health and Tropical Medicine Discipline, College of Public Health, Medical and Veterinary Sciences, Division of Tropical Health and Medicine, James Cook University, Townsville, QLD 4811, Australia; oyelola.adegboye@jcu.edu.au

* Correspondence: aduli.malauaduli@jcu.edu.au; Tel.: +61-747-815-339

Simple Summary: The problem addressed in this research was the possibility of enhancing the nutritional value and health beneficial omega-3 long-chain fatty acid content of lamb and its edible components. The aims and objectives were to evaluate the omega- 3 contents of muscle, liver, kidney, and heart of lot-fed Tattykeel Australian White lambs of the MARGRA brand, in response to dietary supplementation with or without omega-3 oil fortified pellets. The findings demonstrate that the inclusion of omega-3 oil in feedlot diets of lambs enhances the human health beneficial omega-3 longchain polyunsaturated fatty acid profiles of edible muscle tissue and organs without compromising meat quality or shelf life. These results are valuable to society because of increased functionality, health benefits, micro-marbling, tender, mouth-melting taste, and high-end eating quality experience of MARGRA lamb tissues and organs.

Abstract: The aim of this research was to evaluate the nutritional enhancement of omega-3 longchain polyunsaturated fatty acid (n-3 LC-PUFA) composition of edible lamb Longissimus thoracis et lumborum muscle, heart, kidney, and liver in response to dietary supplementation of lot-fed lambs with or without omega-3 oil fortified pellets. The hypothesis tested was that fortifying feedlot pellets with omega-3 oil will enhance the human health beneficial n-3 LC-PUFA composition of edible lamb muscle tissue and organs. Seventy-five Tattykeel Australian White lambs exclusive to the MARGRA brand, with an average body weight of $30 \mathrm{~kg}$ at six months of age, were randomly assigned to the following three dietary treatments of 25 lambs each, and lot-fed as a cohort for 47 days in a completely randomized experimental design: (1) Control grain pellets without oil plus hay; (2) Omega-3 oil fortified grain pellets plus hay; and (3) Commercial whole grain pellets plus hay. All lambs had ad libitum access to the basal hay diet and water. Post-slaughter fatty acid composition of the Longissimus thoracis et lumborum muscle, liver, kidney, and heart were determined using thee gas chromatography-mass spectrophotometry technique. Results indicated significant variations $(p<0.05)$ in fatty acid profiles between tissues and organs. Omega-3 oil fortified pellets significantly $(p<0.05)$ increased $\geq$ C20 n-3 LC-PUFA (C20:5n-3 eicosapentaenoate, EPA + C22:5n3 docosapentaenoate, DPA + C22:6n3 docosahexanoate DHA); C18:3n-3 alpha-linolenate, ALA; C18:2 conjugated linoleic acid, CLA; total monounsaturated fatty acids, MUFA; polyunsaturated fatty acids, PUFA contents; and reduced the ratio of omega- 6 to omega- 3 fatty acids in all lamb organs and tissues without impacting shelf-life. The findings demonstrate that the inclusion of omega-3 oil in 
feedlot diets of lambs enhances the human health beneficial omega-3 long-chain polyunsaturated fatty acid profiles of edible muscle tissue and organs without compromising meat quality.

Keywords: MARGRA lamb; omega-3 fatty acids; Longissimus thoracis et lumborum muscle; kidney; heart; liver; meat quality; oil; whole grain

\section{Introduction}

Functional foods are among the fastest-growing markets in developed countries where the average consumer prefers omega- 3 enrichment with information about the food's production process [1]. Functional foods can influence satiety and a healthier lifestyle [2]. The main strategies for creating healthier and functional foods with increased satiety include the modification of dietary fat, fiber, and sugar compositions [2]. Ansorena and Astiasarán [3] provided insights into the methods of modifying the formulations of fresh, cooked, and fermented meat products in order to increase omega-3 fatty acid content without modifying animal diets. The fortification of functional beef burgers with microencapsulated cod liver oil [4], algal and wheat germ oil emulsions [5] are examples of methods for enriching foods with omega-3 fatty acids.

The demand for high quality meat is on the increase as consumer preferences for edible animal-based protein sources shift toward eating quality with increased human health benefits. Meat, an essential component of the human diet, is rich in nutrients including protein, fatty acids, iron, zinc, copper, selenium, and B-complex vitamins [6-8]. Omega-3 long-chain polyunsaturated fatty acids (n-3 LC-PUFA) are essential fatty acids that play diverse roles in human health and disease prevention. They include the following longer chain derivatives of alpha-linolenic acid (ALA, C18:3n-3): Eicosapentaenoic (EPA, C20:5n-3), docosapentaenoic (DPA, C22:5n-3), and docosahexaenoic (DHA, C22:6n-3) acids. $\mathrm{EPA}+\mathrm{DPA}+\mathrm{DHA}$ are known to promote intellectual development in infancy, relieve inflammation, boost the immune system, reduce incidences of cardiovascular diseases, some cancers, diabetes, allergies, behavioral disorders, and sustain retinal functions [9-12]. However, humans, like all mammals, cannot synthesize n-3 LC-PUFA because they are unable to produce $\Delta 12$ and $\Delta 15$-desaturase enzymes [13], hence, rely on dietary sources like leafy vegetables, oilseeds, nuts, eggs, and seafood, especially fish and crustaceans [10,14], edible marine algae, bacteria, fungi, diatoms, fruits, and herbs [15], to meet their daily n-3 LC-PUFA requirements. Oilseeds commonly used in human diets include rapeseed [16] and soybean [17], while seed oils from waste food by-products such as tomato [18] and citrus [19] are cheap animal feed sources that can enhance healthy fatty acid composition.

Fatty acid composition influences the nutritive value and organoleptic traits of meat including tenderness, flavor, and juiciness [20]. The fatty acid content of meat can be affected by the animal production system [21,22], breed or genotype [23,24], gender [25], age at slaughter [26], liveweight [27], level of fatness [20], type of muscle and feed. In lamb [28], cattle, swine, and poultry [29], it has been suggested that dietary manipulation can be utilized to improve the fatty acid content and nutritional value of meat that more closely meets nutritional guidelines. However, due to extensive rumen microbial biohydrogenation in ruminants, dietary polyunsaturated fatty acids (PUFA) are converted to saturated fatty acids (SFA), absorbed in the small intestine, and deposited in edible tissues (muscles), products (milk), and organs (liver, kidney and heart), thereby causing more health challenges to consumers [30,31]. Ruminant meat research is aimed at reducing saturated fatty acids and increasing the proportion of health-beneficial n-3 LC-PUFA [31]. Therefore, dietary supplementation with rumen-protected plant and fish-based n-3 LCPUFA oil, forages, and concentrates containing bioactively enriched microalgae [32] are some of the steps taken by livestock farmers to improve the nutritional and health values of meat. 
To the current knowledge of the authors of this research, there is presently no published literature on n-3 LC-PUFA metabolism in the Longissimus thoracis et lumborum muscle, heart, kidney, and liver of lot-fed Tattykeel Australian White (TAW) MARGRA lambs in response to dietary supplementation with omega-3 oil. The research reported in this present study intends to fill this knowledge gap. It was hypothesized that fortifying feedlot pellets with omega-3 oil will enhance the human health beneficial n-3 LC-PUFA composition of edible lamb muscle tissue and organs. Therefore, the primary objective of this study was to evaluate and compare the fatty acid profiles in the tissues and organs of TAW lambs raised in a feedlot production system in response to dietary supplementation with or without fortification with omega-3 oil.

\section{Materials and Methods}

\subsection{Animals, Dietary Treatments, and Experimental Design}

This lamb finishing feeding trial was conducted at the Crown Agriculture's feedlot facility at Borenore, New South Wales, Australia, from April to June 2019. Borenore is located at latitude $33^{\circ} 19^{\prime} \mathrm{S}$ and longitude $149^{\circ} 04^{\prime} \mathrm{E}$ with an elevation of 3024 feet above sea level and average annual temperature of $11.7^{\circ} \mathrm{C}\left(53.0^{\circ} \mathrm{F}\right)$ and rainfall of $939.8 \mathrm{~mm}$ ( 37.0 inches). The feedlot was an automated facility in a well-ventilated covered building with concrete floors, density of five square meters per head with all the feeding troughs equipped with installed sensors capable of immediate data capture of each lamb's ear tag identification, entry and exit times, body weight, feed intake, and other vital parameters. These data are automatically recorded, electronically cloud-stored, and directly downloadable into Excel spreadsheets and transmitted when required. Seventy-five Tattykeel Australian White lambs exclusive to the MARGRA brand, with an average body weight of $30 \mathrm{~kg}$ at six months of age, were randomly assigned to the following three dietary treatments of 25 lambs each, and lot-fed as a cohort for 47 days after a 14-day adaptation period in a completely randomized experimental design: (1) Control grain pellets without oil plus hay; (2) omega-3 oil fortified grain pellets plus hay; and (3) commercial whole grain pellets plus hay. All lambs had ad libitum access to the basal hay diet and water. The nutrient composition of the supplementary and basal diets is presented in Table 1.

Table 1. Nutrient composition of the control, n-3 LC PUFA, MSM whole grain, and hay feeds \#.

\begin{tabular}{ccccc}
\hline \multirow{2}{*}{ Nutrient Composition (\%DM) } & \multicolumn{4}{c}{ Experimental and Basal Diets } \\
\cline { 2 - 5 } & Control & Omega-3 & MSM Whole Grain & Hay \\
\hline Dry Matter (DM) & 90.8 & 91.7 & 90.3 & 93.4 \\
Moisture & 9.2 & 8.3 & 9.7 & 6.6 \\
Acid Detergent Fiber (ADF) & 7.6 & 8.2 & 6.3 & 39.4 \\
Neutral Detergent Fiber (NDF) & 23.8 & 23.0 & 16.8 & 7.9 \\
Crude Protein (CP) & 16.9 & 8.0 & 7.2 & 8.1 \\
Ash & 7.8 & 10.3 & 6.0 & 3.3 \\
Ether Extract (EE) & 6.1 & 15.1 & 14.4 & 8.3 \\
Metabolizable Energy (ME) MJ/kg & 14.1 & 83.8 & 87.5 & 46.8 \\
Dry Matter Digestibility (DMD) & 84.9 & 82.6 & 86.2 & 47.1 \\
Digestible Organic Matter (DOMD) & 83.7 & & \\
\hline
\end{tabular}

\# DOMD, Digestible organic matter in the dry matter; TDN, \%Total digestible nutrients (as \% of DM) $=82.38-(0.7515 \times \mathrm{ADF}[\%$ of $\mathrm{DM}]$ ); $\mathrm{ME}$, Metabolizable energy $(\mathrm{DE}[\mathrm{Mcal} / \mathrm{kg}]=\% \mathrm{TDN} \times 0.01 \times 4.4)$ where $\mathrm{DE}$ is digestible energy, which was converted as $\mathrm{ME}=(\mathrm{DE}(\mathrm{Mcal} / \mathrm{kg}) \times 0.82) \times 4.185$.

At the end of the feeding trial, the lambs were conveyed during the cool hours of the day to the Gundagai Meat Processing Plant, New South Wales, Australia, held in lairage and fasted overnight. The lambs were humanely sacrificed as a single mob in line with Meat Standards Australia guidelines and industry best practice standards. The carcasses were subjected to medium voltage electrical stimulation before being trimmed and dressed [33]. The liver, kidney, and heart were sampled immediately following evisceration, vacuumsealed in labelled bags, and stored at $-20{ }^{\circ} \mathrm{C}$ pending fatty acid evaluation. All carcasses 
were held in the chiller room for $24 \mathrm{~h}$ at $4{ }^{\circ} \mathrm{C}$ and a sample of the Longissimus thoracis et lumborum muscle tissue was taken between the 12th and 13th ribs for fatty acid analysis.

\subsection{Feed Sample Processing and Nutrient Composition Analysis}

Supplementary and basal feed samples were oven-dried for three days at $60{ }^{\circ} \mathrm{C}$, cooled, and ground to pass through a $1 \mathrm{~mm}$ sieve using a laboratory mill (Thomas Model 4 Wiley ${ }^{\circledR}$ Mill; Thomas Scientific, Swedesboro, NJ, USA). Dry matter and ash percentages were determined using the AOAC standard laboratory analytical techniques [34]. Neutral detergent (NDF) and acid detergent (ADF) fiber percentages were determined using an Ankom Fiber Analyzer (ANKOM2000; ANKOM Technology, Macedon, NY, USA). Nitrogen content was determined using a Thermo Finnigan EA 1112 Series Flash Elemental Analyzer (Thermo Finnigan, Poway, CA, USA) and the values were multiplied by 6.25 to provide the expected crude protein $(\mathrm{CP})$ percentage. Ether extract (EE) was analyzed employing an ANKOM $^{\mathrm{XT15}}$ fat/oil extractor (ANKOM Technology, Macedon, NY, USA).

\subsection{Fatty Acid Analysis}

Fatty acid analysis of feed, muscle tissue, liver, kidney, and heart samples was carried out at the Commonwealth Scientific and Industrial Research Organization (CSIRO), Food Nutrition and Bio-based Products, Oceans and Atmosphere Laboratory, Hobart, Tasmania, Australia. The gas chromatography-mass spectrophotometry total lipids and muscle phospholipids extraction procedures of Malau-Aduli et al. [35,36] based on an amended Bligh and Dyer technique [37], were utilized for fatty acid composition analysis where total lipids in $1 \mathrm{~g}$ of un-homogenized muscle tissue samples were extracted overnight. The original phase was a single-phase overnight extraction utilizing $\mathrm{CHCl}_{3}: \mathrm{MeOH}: \mathrm{H}_{2} \mathrm{O}$ (1:2:0.8 $v / v)$. The second segment involved phase separation with the addition of $\mathrm{CHCl}_{3}$ :saline Milli-Q $\mathrm{H}_{2} \mathrm{O}(1: 1 \mathrm{v} / \mathrm{v})$ followed by rotary evaporation of the lower chloroform phase at $40{ }^{\circ} \mathrm{C}$ to acquire total lipids. The extracted cumulative lipids were separated into lipid classes by thin-layer chromatography (TLC) using $100 \mathrm{~mL}$ of the lipid extract reconstituted in n-hexane. The extract was marked onto silica gel G plates $\left(200 \times 200 \times 0.25 \mathrm{~mm}^{3}\right)$ using a micropipette. The TLC plate was developed in an acetone/petroleum ether (1:3 vol/vol) solvent system in a tank comprising a few crystals of butylated hydroxytoluene (BHT) to hinder oxidation. Triacylglycerols, cholesterol, and free fatty acids migrated, while phospholipids remained at the origin of the plate. The phospholipids were scraped off the plate into clean screw-capped test tubes for transmethylation and eventual computation of the lipid conversion factor (LCF) of 0.912 based on g fatty acids/g total lipids ( 0.083 for phospholipids, 0.829 for triacylglycerols, and $0 \%$ for cholesterol since cholesterol does not have any fatty acids). An aliquot from each total lipid extract was utilized for transmethylation with $\mathrm{MeOH}: \mathrm{CHCl}_{3}: \mathrm{HCl}(10: 1: 1 \mathrm{v} / \mathrm{v})$ for two hours at $80^{\circ} \mathrm{C}$. Fatty acid methyl esters (FAME) were extracted thrice using n-hexane: $\mathrm{CHCl}_{3}(4: 1 v / v)$. A known concentration of an internal standard (C19:0) was added in a $1500 \mu \mathrm{L}$ vial encompassing the extracted FAME. The FAME was analyzed on a 7890B gas chromatograph (Agilent Technologies, Palo Alto, CA, USA) furnished with an Equity ${ }^{\mathrm{TM}}-1$ fused $15 \mathrm{~m}$ silica capillary column with $0.1 \mathrm{~mm}$ internal diameter and $0.1 \mu \mathrm{m}$ film thickness (Supelco, Bellefonte, PA, USA), a flame ionization sensor, a split/splitless injector, and an Agilent Technologies $7683 \mathrm{~B}$ Series autosampler. The gas chromatograph settings were splitless mode injection; carrier gas He; original oven temperature $120^{\circ} \mathrm{C}$ and then increased to $270{ }^{\circ} \mathrm{C}$ at flow rates of $10{ }^{\circ} \mathrm{C} / \mathrm{min}$ and to $310^{\circ} \mathrm{C}$ at $5{ }^{\circ} \mathrm{C} / \mathrm{min}$. The Agilent Technologies ChemStation software (Palo Alto, CA, USA) was used to measure fatty acid peaks. The fatty acid identities were established using a Finnigan Thermoquest GCQTM GC/MS fitted with an on-column injector and Thermoquest Xcalibur software (Austin, TX, USA) as described in detail by Miller et al. [38]. Fatty acid percentages were calculated as follows: FA $\%=[($ individual fatty acid area) $\times(100)] /$ (sum total area of fatty acids). Fatty acid contents were calculated as follows: FA mg $/ 100 \mathrm{~g}=($ Total lipid $) \times(\mathrm{LCF}[0.912]) \times([\% \mathrm{FA}] / 100) \times 1000$, where 0.912 
was the resultant lipid conversion factor [39]. FA contents were presented in mg/100 g tissue as per Food Standards of Australia and New Zealand recommendations.

\subsection{Statistical Analyses}

Data analysis was performed as a completely randomized design using $\mathrm{R}$ statistical software version 3.6.3 [40]. Statistical inference was based on a 5\% level of significance. Summary statistics of fatty acids composition were presented as means and standard deviations. The effect of dietary treatment was statistically analyzed separately using one-way analysis of variance (ANOVA) in the general linear model (GLM) procedure to investigate the fatty acid profile differences in the muscle, liver, kidney, and heart of the TAW lambs.

The model utilized was:

$$
F A_{i j}=\mu+\text { Feed }_{i}+\epsilon_{i j}
$$

where $F A$ is the fatty acid composition; $\mu$ is the overall mean response; Feed ${ }_{i}$ is the effect due to the $i$ th treatment ( $i=1$ to 3 ; control, omega-3, MSM whole grain); and $\epsilon_{i j}$ is the random error. Dunn's post-hoc test [41,42] for multiple comparisons of groups with Hochberg's adjustment $[43,44]$ was used to further examine which treatment was responsible for the differences among means of fatty acids that were statistically significant in the oneway ANOVA.

\section{Results}

\subsection{Fatty Acid Composition of Basal and Supplementary Feeds}

The fatty acid profiles of the basal (hay), control (without oil), omega-3 oil-fortified (omega-3), and whole grain (MSM) pelleted diets are shown in Table 2. All the supplementary diets were formulated to be isocaloric (metabolizable energy of $14-15 \mathrm{MJ} / \mathrm{kg}$ ) and isonitrogenous (crude protein of $16.4-17.0 \%$ ) with a dry matter digestibility of $83.8-87.5 \%$. As depicted in Table 1, the omega-3 oil-infused diet had higher EPA + DHA + DPA, n-3 LC-PUFA and ALA $(2.74,19.18$, and $15.39 \mathrm{mg} / 100 \mathrm{~g}$, respectively), and lower total n-6 PUFA and ratios of n-6/n-3 and PUFA/SFA (28.16, 1.47, and $0.75 \mathrm{mg} / 100 \mathrm{~g}$, respectively) than the control and MSM whole grain diets. The MSM whole grain diet had the highest proportions of C18:2n-6 (linoleic acid) and oleic acid (C18:1) (92.38 and 75.06 mg/100 g, respectively). The control diet had a higher $n-6 / n-3$ ratio value of 9.08 , while the basal hay diet had the highest proportion of C20:2n-6 and C20:4n-6 (arachidonic acid).

\subsection{Fatty Acid Profile of the Longissimus thoracis et lumborum Muscle}

The fatty acid composition of the longissimus thoracis et lumborum muscle tissue is presented in Table 3. The omega-3 oil diet produced lamb muscles with the highest contents of n-3 LC-PUFA, DHA, EPA, DPA, C18:3n-3, C18:1, C18:0 (stearic acid), total SFA, MUFA, and PUFA/SFA ratio. The MSM whole grain diet produced muscles with the highest n-6/n-3 PUFA ratio. A boxplot of Hochberg's adjusted multiple comparisons of significant differences between the treatment groups in muscle fatty acid profiles are depicted in Figure 1, where the omega-3 diet consistently shows a significantly higher fatty acid concentration than the control and MSM whole grain diets.

\subsection{Fatty Acid Content of Liver}

In the liver (Table 4), it was evident that the sheer volume of total fatty acid metabolism output was greater than in the muscle, kidney, and heart. The omega- 3 oil diet had significantly higher CLA $(p<0.0346)$, EPA + DHA $(p<0.0000)$, EPA + DHA + DPA $(p<0.0002)$, PUFA/SFA $(p<0.0365)$, n-3 LC-PUFA $(p<0.0004)$, n-6 PUFA $(p<0.0008)$, and $\mathrm{n}-6 / \mathrm{n}-3$ PUFA ratio $(p<0.0000)$ than the other treatment groups. A boxplot of Hochberg's adjusted multiple comparisons of significant differences between the treatment groups in liver fatty acid profiles are depicted in Figure 2, where the omega-3 oil diet maintained a significantly higher fatty acid content than the control and MSM whole grain diets. However, the compositions of C14:0, C17:1n8c + a17:0, C18:3n-6, C19:1, C20:4n-6, C20:3, 
CLA, C22:5n-6, C22:4n-6, C23:0, n-6 PUFA, and n-6/n-3 PUFA ratio in the MSM whole grain diet were higher than in the control and omega- 3 oil diets.

Table 2. Fatty acid composition (mg/100 g) of basal and supplementary diets \#.

\begin{tabular}{|c|c|c|c|c|}
\hline Fatty Acid & Omega-3 & Control & MSM Whole Grain & Basal Hay \\
\hline $13: 0$ & 0.00 & 0.00 & 0.01 & 0.00 \\
\hline $14: 1$ & 0.00 & 0.00 & 0.00 & 0.00 \\
\hline 14:0 & 2.23 & 0.45 & 0.25 & 0.33 \\
\hline $15: 0$ & 2.62 & 0.36 & 0.30 & 0.21 \\
\hline $16: 1$ & 1.02 & 0.79 & 1.05 & 1.88 \\
\hline $16: 0$ & 35.96 & 27.19 & 30.52 & 12.53 \\
\hline $17: 1 \mathrm{n} 8 \mathrm{c}+\mathrm{a} 17: 0$ & 0.64 & 0.27 & 0.25 & 0.18 \\
\hline $17: 0$ & 1.09 & 0.33 & 0.30 & 0.13 \\
\hline $18: 3 n 6$ & 0.32 & 0.00 & 0.00 & 0.24 \\
\hline $18: 4 n 3$ & 0.32 & 0.00 & 0.00 & 0.20 \\
\hline 18:2n6 (LA) & 25.25 & 81.75 & 92.38 & 30.02 \\
\hline 18:3n3 (ALA) & 15.39 & 8.84 & 11.21 & 6.13 \\
\hline CLA & 0.65 & 0.10 & 0.29 & 0.00 \\
\hline $18: 0$ & 8.25 & 4.59 & 4.13 & 2.74 \\
\hline $18: 1$ & 31.92 & 57.34 & 75.06 & 51.23 \\
\hline 19:1 & 0.00 & 0.04 & 0.06 & 0.04 \\
\hline 20:4n6 (ARA) & 0.00 & 0.00 & 0.00 & 0.53 \\
\hline 20:5n3 (EPA) & 0.22 & 0.18 & 0.23 & 0.00 \\
\hline $20: 3$ & 0.00 & 0.00 & 0.01 & 0.00 \\
\hline $20: 3 n 6$ & 0.80 & 0.15 & 0.16 & 0.18 \\
\hline $20: 4 n 3$ & 0.30 & 0.00 & 0.00 & 0.02 \\
\hline $20: 2 n 6$ & 0.32 & 0.13 & 0.21 & 0.33 \\
\hline $20: 1$ & 1.51 & 1.71 & 2.02 & 1.79 \\
\hline 20:0 & 2.80 & 0.60 & 0.81 & 0.42 \\
\hline $21: 5 \mathrm{n} 3$ & 0.43 & 0.00 & 0.00 & 0.00 \\
\hline $21: 0$ & 1.17 & 0.16 & 0.08 & 0.00 \\
\hline $22: 5 n 6$ & 0.87 & 0.01 & 0.08 & 0.00 \\
\hline 22:6n3 (DHA) & 1.53 & 0.03 & 0.05 & 0.93 \\
\hline $22: 4 n 6$ & 0.60 & 0.08 & 0.05 & 0.00 \\
\hline 22:5n3 (DPA) & 0.99 & 0.00 & 0.17 & 0.22 \\
\hline $22: 1$ & 2.39 & 0.47 & 0.34 & 0.25 \\
\hline $22: 0$ & 4.63 & 0.44 & 0.60 & 0.23 \\
\hline $23: 1$ & 0.64 & 0.06 & 0.00 & 0.00 \\
\hline $23: 0$ & 1.72 & 0.01 & 0.14 & 0.02 \\
\hline $24: 1$ & 0.94 & 0.25 & 0.28 & 0.14 \\
\hline 24:0 & 3.54 & 0.40 & 0.54 & 0.15 \\
\hline Total FA & 147.11 & 185.91 & 220.96 & 110.69 \\
\hline $\mathrm{EPA}+\mathrm{DHA}$ & 1.74 & 0.21 & 0.28 & 0.93 \\
\hline $\mathrm{EPA}+\mathrm{DHA}+\mathrm{DPA}$ & 2.74 & 0.21 & 0.45 & 1.16 \\
\hline SFA & 64.02 & 34.52 & 37.66 & 16.77 \\
\hline MUFA & 35.09 & 60.13 & 78.46 & 55.12 \\
\hline PUFA & 48.00 & 91.26 & 104.84 & 38.81 \\
\hline PUFA/SFA & 0.75 & 2.64 & 2.78 & 2.31 \\
\hline$\sum n 3 P U F A$ & 19.18 & 9.05 & 11.66 & 7.51 \\
\hline$\sum$ n6PUFA & 28.16 & 82.12 & 92.88 & 31.30 \\
\hline n6/n3PUFA & 1.47 & 9.08 & 7.97 & 4.17 \\
\hline
\end{tabular}

\# LA, linoleic acid; ALA, $\alpha$-linolenic acid; CLA, conjugated linolenic acid; ARA, arachidonic acid; EPA, eicosapentaenoic acid; DHA, docosahexaenoic acid; DPA, docosapentaenoic acid; $\Sigma$ SFA, total saturated fatty acids; FA, fatty acid; $\Sigma$ MUFA, total monounsaturated fatty acids; and total polyunsaturated fatty acids ( $\Sigma$ PUFA); $\sum$ SFA is the sum of 14:0, 15:0, 16:0, 17:0, 18:0, 20:0, 21:0, 22:0, 23:0, 24:0; $\sum$ MUFA is the sum of 14:1, 16:1, 17:1n-8 + a17:0, 18:1, 19:1, 20:1, 22:1, 23:1, 24:1; $\sum$ PUFA is the sum of 18:4n-3, 18:3n-6, 18:2n-6, 18:3n-3, 20:3, 20:4n-3, 20:4n-6, 20:5n-3, 20:3n-6, 20:2n-6, 22:6n-3, 22:5n-3, 22:5n-6, 22:4n-6; $\sum n-3$ LC-PUFA is the sum of 18:3n-3, 18:4n-3, 20:4n-3, 20:5n-3, 22:6n-3, $22: 5 n-3 ; \sum n-6$ PUFA is the sum of $18: 2 n-6,18: 3 n-6,20: 4 n-6,20: 3 n-6,20: 2 n-6,22: 5 n-6,22: 4 n-6$. 
Table 3. Fatty acid profile (mg/100 g) of Longissimus thoracis et lumborum muscle tissue in TAW lambs \#.

\begin{tabular}{|c|c|c|c|c|}
\hline Fatty Acid & Omega-3 & Control & MSM Whole Grain & $p$-Value \\
\hline 13:0 & $0.05 \pm 0.11$ & $0.03 \pm 0.04$ & $0.00 \pm 0.00$ & 0.1108 \\
\hline 14:1 & $0.63 \pm 0.65$ & $0.31 \pm 0.33$ & $0.19 \pm 0.34$ & 0.0417 \\
\hline 14:0 & $24.25 \pm 18.62$ & $16.80 \pm 7.84$ & $14.22 \pm 8.06$ & 0.0809 \\
\hline $15: 1$ & $4.44 \pm 2.36$ & $2.26 \pm 1.22$ & $1.58 \pm 0.85$ & 0.0005 \\
\hline $16: 0$ & $320.50 \pm 182.90$ & $209.83 \pm 83.05$ & $169.06 \pm 79.43$ & 0.0106 \\
\hline $16: 1$ & $25.86 \pm 17.40$ & $16.93 \pm 7.29$ & $14.43 \pm 8.57$ & 0.0398 \\
\hline $17: 1 \mathrm{n} 8 \mathrm{c}+\mathrm{a} 17: 0$ & $13.83 \pm 7.99$ & $9.40 \pm 3.68$ & $7.85 \pm 3.55$ & 0.0201 \\
\hline $17: 0$ & $16.85 \pm 9.12$ & $10.80 \pm 4.08$ & $8.31 \pm 3.10$ & 0.0035 \\
\hline $18: 3 n 6$ & $1.09 \pm 0.38$ & $0.93 \pm 0.28$ & $0.71 \pm 0.31$ & 0.0116 \\
\hline $18: 4 n-3$ & $0.34 \pm 0.28$ & $0.01 \pm 0.04$ & $0.03 \pm 0.05$ & 0.0006 \\
\hline 18:2n-6 (LA) & $99.35 \pm 25.12$ & $57.06 \pm 14.44$ & $46.89 \pm 10.66$ & 0.0000 \\
\hline 18:3n-3 (ALA) & $15.70 \pm 5.01$ & $8.76 \pm 2.46$ & $6.87 \pm 2.64$ & 0.0000 \\
\hline CLA & $3.83 \pm 2.05$ & $2.65 \pm 1.03$ & $1.79 \pm 0.93$ & 0.0030 \\
\hline 18:0 & $208.83 \pm 119.80$ & $139.11 \pm 54.40$ & $99.63 \pm 40.82$ & 0.0042 \\
\hline $18: 1$ & $582.31 \pm 364.95$ & $395.67 \pm 173.74$ & $305.06 \pm 147.93$ & 0.0174 \\
\hline 19:1 & $1.31 \pm 0.68$ & $0.89 \pm 0.37$ & $0.93 \pm 0.51$ & 0.1222 \\
\hline 20:4n-6 (ARA) & $21.87 \pm 8.20$ & $11.26 \pm 8.18$ & $4.78 \pm 3.64$ & 0.0000 \\
\hline $20: 5 n-3$ (EPA) & $9.68 \pm 3.68$ & $4.25 \pm 2.15$ & $2.36 \pm 1.01$ & 0.0000 \\
\hline $22: 3$ & $0.19 \pm 0.31$ & $0.21 \pm 0.22$ & $0.18 \pm 0.23$ & 0.9168 \\
\hline $20: 3 n-6$ & $4.61 \pm 1.17$ & $2.10 \pm 0.68$ & $1.74 \pm 0.58$ & 0.0000 \\
\hline $20: 4 n-3$ & $0.02 \pm 0.05$ & $0.10 \pm 0.18$ & $0.15 \pm 0.24$ & 0.0945 \\
\hline $20: 2 n-6$ & $1.66 \pm 0.41$ & $0.52 \pm 0.20$ & $0.45 \pm 0.29$ & 0.0000 \\
\hline 20:0 & $1.71 \pm 1.01$ & $1.15 \pm 0.40$ & $0.82 \pm 0.37$ & 0.0051 \\
\hline 20:1 & $4.45 \pm 2.34$ & $1.71 \pm 0.63$ & $1.30 \pm 0.68$ & 0.0001 \\
\hline $21: 5 n-3$ & $0.15 \pm 0.13$ & $0.14 \pm 0.12$ & $0.11 \pm 0.12$ & 0.4905 \\
\hline 21:0 & $0.13 \pm 0.22$ & $0.04 \pm 0.09$ & $0.10 \pm 0.14$ & 0.5992 \\
\hline $22: 5 n-6$ & $0.27 \pm 0.22$ & $0.26 \pm 0.25$ & $0.24 \pm 0.11$ & 0.6978 \\
\hline 22:6n-3 (DHA) & $5.59 \pm 1.63$ & $1.85 \pm 0.81$ & $1.28 \pm 0.67$ & 0.0000 \\
\hline $22: 4 n-6$ & $1.10 \pm 0.39$ & $0.93 \pm 0.33$ & $0.91 \pm 0.25$ & 0.2010 \\
\hline 22:5n-3 (DPA) & $9.69 \pm 3.36$ & $5.48 \pm 1.75$ & $4.42 \pm 1.25$ & 0.0000 \\
\hline 22:0 & $0.48 \pm 0.27$ & $0.34 \pm 0.17$ & $0.36 \pm 0.43$ & 0.3908 \\
\hline $22: 1$ & $0.68 \pm 0.31$ & $0.52 \pm 0.14$ & $0.43 \pm 0.21$ & 0.0218 \\
\hline $23: 1$ & $0.00 \pm 0.00$ & $0.00 \pm 0.00$ & $0.02 \pm 0.05$ & 0.2268 \\
\hline 23:0 & $0.78 \pm 0.18$ & $0.52 \pm 0.16$ & $0.41 \pm 0.09$ & 0.0000 \\
\hline $24: 1$ & $1.37 \pm 0.33$ & $0.66 \pm 0.16$ & $0.66 \pm 0.24$ & 0.0000 \\
\hline 24:0 & $0.98 \pm 0.29$ & $0.61 \pm 0.17$ & $0.53 \pm 0.25$ & 0.0004 \\
\hline Total FA & $1384.60 \pm 766.56$ & $904.11 \pm 342.20$ & $698.78 \pm 307.88$ & 0.0056 \\
\hline EPA + DHA & $15.28 \pm 5.12$ & $6.11 \pm 2.88$ & $3.64 \pm 1.49$ & 0.0000 \\
\hline $\mathrm{EPA}+\mathrm{DHA}+\mathrm{DPA}$ & $24.97 \pm 8.27$ & $11.58 \pm 4.54$ & $8.05 \pm 2.56$ & 0.0000 \\
\hline$\sum \mathrm{SFA}$ & $579.40 \pm 333.52$ & $381.87 \pm 149.39$ & $295.27 \pm 131.95$ & 0.0079 \\
\hline$\sum$ MUFA & $630.23 \pm 393.61$ & $425.92 \pm 185.28$ & $330.79 \pm 161.51$ & 0.0172 \\
\hline$\sum$ PUFA & $175.14 \pm 47.59$ & $96.53 \pm 28.59$ & $72.90 \pm 19.03$ & 0.0000 \\
\hline PUFA/SFA & $0.36 \pm 0.14$ & $0.29 \pm 0.12$ & $0.27 \pm 0.10$ & 0.1210 \\
\hline$\sum n-3 P U F A$ & $41.36 \pm 12.83$ & $20.81 \pm 6.43$ & $15.38 \pm 5.24$ & 0.0000 \\
\hline$\sum$ n-6PUFA & $133.78 \pm 35.37$ & $75.71 \pm 22.91$ & $57.51 \pm 14.31$ & 0.0000 \\
\hline n-6/n-3PUFA & $3.33 \pm 0.52$ & $3.72 \pm 0.63$ & $3.87 \pm 0.65$ & 0.0499 \\
\hline
\end{tabular}

\# Abbreviations as in Table 2. 


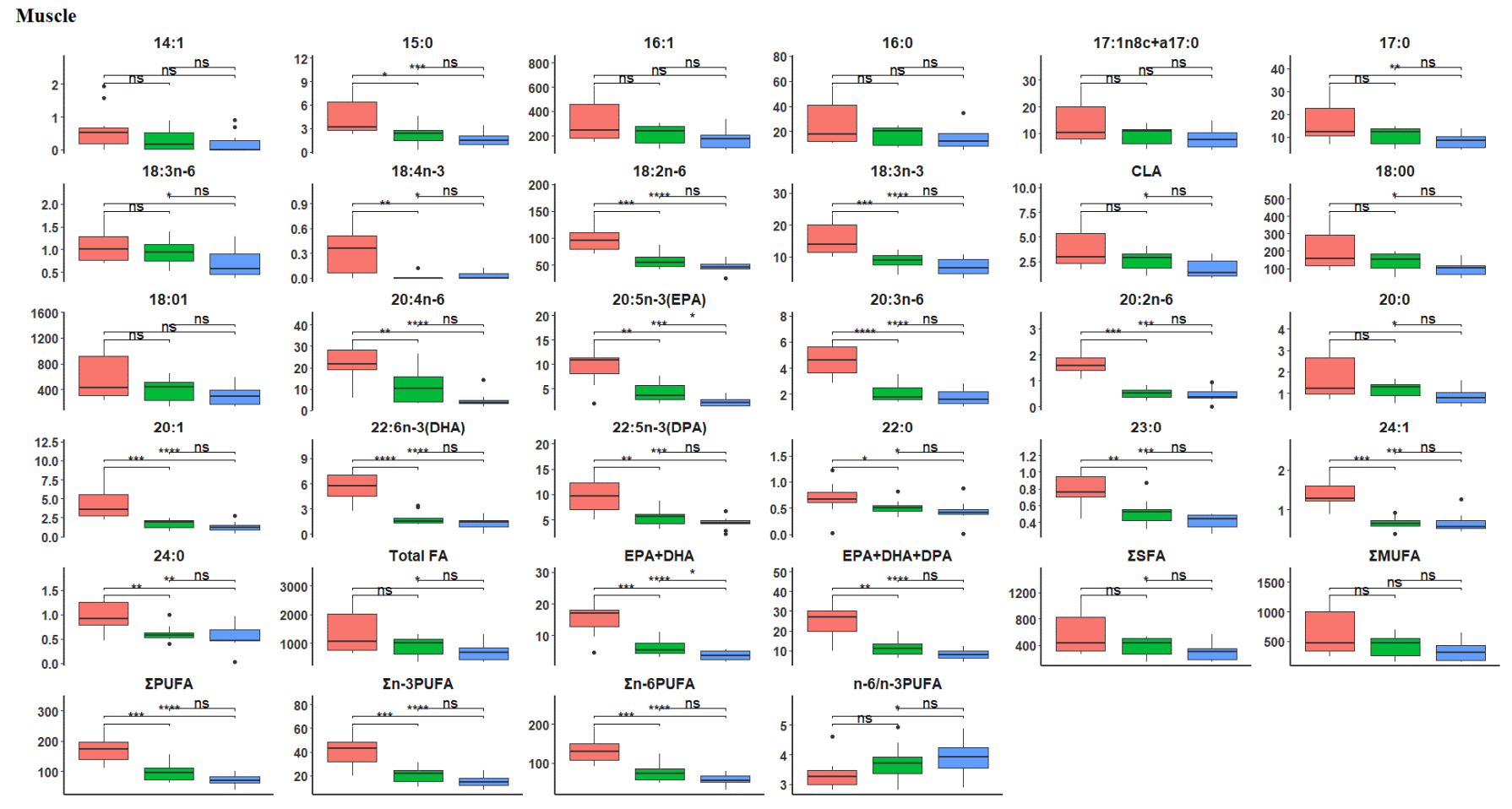

宇 Omega control MSM

Figure 1. Boxplots showing the distribution of selected fatty acid composition in the muscle tissue. Each plot tested the mean fatty acid in omega-3 versus control, omega-3 versus MSM whole grain, and control versus MSM whole grain with Hochberg's adjusted multiple comparisons. ${ }^{*} p<0.05 ;{ }^{* *} p<0.01$; ${ }^{* * *} p<0.001 ;{ }^{* * *} p<0.0001$; ns, not significant $(p>0.05)$.

Liver
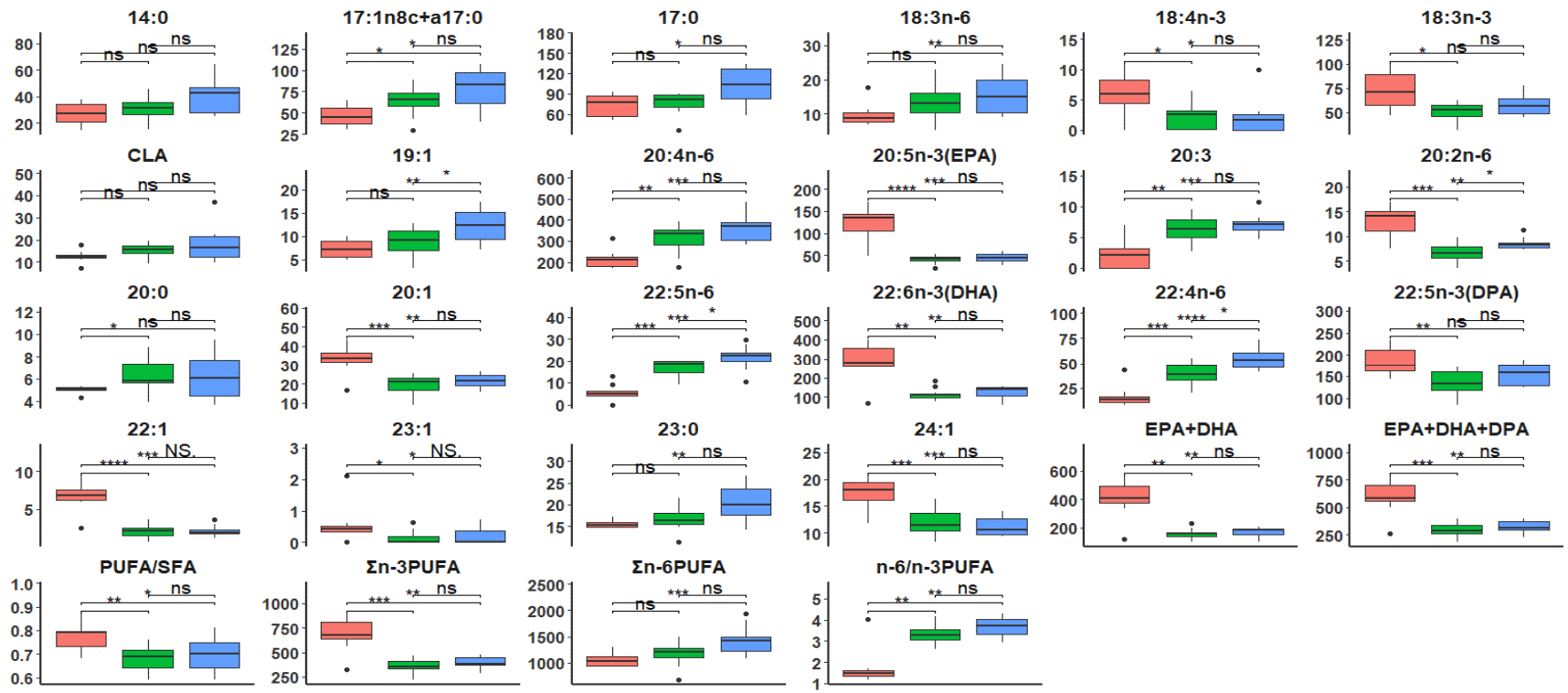

Omega Control MSM

Figure 2. Boxplots showing the distribution of selected fatty acids composition in the liver. Each plot tested the mean fatty acid in omega-3 versus control, omega-3 versus MSM whole grain, and control versus MSM whole grain with Hochberg's adjusted multiple comparisons. ${ }^{*} p<0.05{ }^{* *} p<0.01{ }^{* * *} p<0.001 ;{ }^{* * * *} p<0.0001$; ns, not significant $(p>0.05)$. 
Table 4. Fatty acid profile (mg/100 g) of the liver in TAW lambs\#.

\begin{tabular}{|c|c|c|c|c|}
\hline Fatty Acid & Omega-3 & Control & MSM Whole Grain & $p$-Value \\
\hline $13: 0$ & $0.24 \pm 0.26$ & $0.16 \pm 0.26$ & $0.36 \pm 0.50$ & 0.3890 \\
\hline $14: 1$ & $0.84 \pm 0.92$ & $1.45 \pm 1.21$ & $0.55 \pm 0.86$ & 0.6411 \\
\hline $14: 0$ & $26.95 \pm 8.36$ & $31.22 \pm 8.74$ & $40.09 \pm 13.89$ & 0.0190 \\
\hline $15: 1$ & $19.20 \pm 6.55$ & $15.49 \pm 5.47$ & $18.81 \pm 7.39$ & 0.7370 \\
\hline $16: 0$ & $755.15 \pm 151.51$ & $765.95 \pm 134.32$ & $906.84 \pm 205.56$ & 0.0938 \\
\hline $16: 1$ & $66.47 \pm 18.67$ & $83.97 \pm 26.54$ & $89.50 \pm 28.94$ & 0.0783 \\
\hline $17: 1 \mathrm{n} 8 \mathrm{c}+\mathrm{a} 17: 0$ & $46.43 \pm 11.84$ & $62.51 \pm 16.78$ & $78.31 \pm 25.78$ & 0.0017 \\
\hline $17: 0$ & $73.54 \pm 16.08$ & $76.10 \pm 16.51$ & $101.28 \pm 26.67$ & 0.0143 \\
\hline $18: 3 n 6$ & $9.67 \pm 3.17$ & $13.31 \pm 4.89$ & $15.45 \pm 5.60$ & 0.0160 \\
\hline $18: 4 n-3$ & $6.10 \pm 3.34$ & $2.34 \pm 2.22$ & $2.15 \pm 3.18$ & 0.0098 \\
\hline $18: 2 n-6(L A)$ & $508.28 \pm 68.59$ & $438.04 \pm 84.82$ & $570.01 \pm 99.23$ & 0.2330 \\
\hline 18:3n-3 (ALA) & $72.78 \pm 18.68$ & $50.46 \pm 9.52$ & $58.65 \pm 12.48$ & 0.0655 \\
\hline CLA & $12.45 \pm 2.57$ & $15.13 \pm 3.13$ & $18.05 \pm 8.47$ & 0.0346 \\
\hline 18:0 & $1050.30 \pm 82.46$ & $879.11 \pm 139.95$ & $1058.24 \pm 190.38$ & 0.9770 \\
\hline $18: 1$ & $1414.42 \pm 210.44$ & $1414.52 \pm 268.81$ & $1521.50 \pm 345.97$ & 0.4875 \\
\hline $19: 1$ & $7.25 \pm 1.88$ & $8.93 \pm 2.98$ & $12.35 \pm 3.47$ & 0.0010 \\
\hline $20: 4 n-6$ (ARA) & $213.71 \pm 41.72$ & $310.53 \pm 69.00$ & $368.35 \pm 71.25$ & 0.0000 \\
\hline 20:5n-3 (EPA) & $122.60 \pm 36.37$ & $40.03 \pm 9.65$ & $43.99 \pm 10.29$ & 0.0000 \\
\hline $22: 3$ & $2.08 \pm 2.23$ & $6.35 \pm 2.14$ & $7.16 \pm 1.72$ & 0.0001 \\
\hline $20: 3 n-6$ & $58.65 \pm 9.41$ & $33.88 \pm 5.74$ & $49.26 \pm 16.35$ & 0.1700 \\
\hline $20: 4 n-3$ & $6.07 \pm 0.84$ & $5.00 \pm 2.59$ & $6.03 \pm 1.75$ & 0.9550 \\
\hline $20: 2 n-6$ & $12.96 \pm 3.02$ & $6.72 \pm 1.79$ & $8.59 \pm 1.24$ & 0.0033 \\
\hline 20:0 & $33.42 \pm 7.26$ & $19.28 \pm 5.06$ & $21.45 \pm 3.60$ & 0.0652 \\
\hline 20:1 & $5.02 \pm 0.28$ & $6.25 \pm 1.61$ & $6.38 \pm 1.97$ & 0.0007 \\
\hline $21: 5 n-3$ & $1.58 \pm 0.71$ & $3.27 \pm 2.79$ & $2.76 \pm 2.20$ & 0.2770 \\
\hline 21:0 & $0.37 \pm 0.21$ & $0.28 \pm 0.29$ & $0.51 \pm 0.26$ & 0.2643 \\
\hline $22: 5 n-6$ & $5.23 \pm 3.89$ & $17.08 \pm 3.63$ & $21.53 \pm 5.93$ & 0.0000 \\
\hline 22:6n-3 (DHA) & $286.77 \pm 95.79$ & $116.09 \pm 30.92$ & $124.56 \pm 30.70$ & 0.0001 \\
\hline $22: 4 n-6$ & $16.44 \pm 9.99$ & $39.84 \pm 11.41$ & $54.47 \pm 11.01$ & 0.0000 \\
\hline 22:5n-3 (DPA) & $185.12 \pm 30.84$ & $133.09 \pm 29.99$ & $154.08 \pm 24.89$ & 0.0515 \\
\hline $22: 0$ & $6.78 \pm 1.87$ & $2.05 \pm 0.87$ & $2.12 \pm 0.74$ & 0.0000 \\
\hline $22: 1$ & $9.76 \pm 0.84$ & $8.20 \pm 1.11$ & $9.31 \pm 1.81$ & 0.5057 \\
\hline $23: 1$ & $0.56 \pm 0.57$ & $0.14 \pm 0.22$ & $0.17 \pm 0.27$ & 0.0504 \\
\hline $23: 0$ & $15.48 \pm 0.82$ & $16.69 \pm 2.77$ & $20.36 \pm 4.07$ & 0.0011 \\
\hline $24: 1$ & $17.44 \pm 2.66$ & $11.81 \pm 2.62$ & $11.14 \pm 1.71$ & 0.0000 \\
\hline 24:0 & $16.10 \pm 1.00$ & $15.49 \pm 2.52$ & $17.48 \pm 2.82$ & 0.2397 \\
\hline Total FA & $5085.02 \pm 632.20$ & $4650.60 \pm 806.74$ & $5421.65 \pm 1044.59$ & 0.5016 \\
\hline $\mathrm{EPA}+\mathrm{DHA}$ & $409.37 \pm 127.95$ & $156.12 \pm 37.43$ & $168.54 \pm 35.69$ & 0.0000 \\
\hline $\mathrm{EPA}+\mathrm{DHA}+\mathrm{DPA}$ & $594.49 \pm 153.14$ & $289.22 \pm 63.49$ & $322.62 \pm 56.93$ & 0.0002 \\
\hline$\sum \mathrm{SFA}$ & $1972.10 \pm 229.59$ & $1814.93 \pm 295.51$ & $2179.67 \pm 431.38$ & 0.2769 \\
\hline$\sum M U F A$ & $1593.04 \pm 242.26$ & $1604.51 \pm 317.62$ & $1736.91 \pm 404.01$ & 0.4181 \\
\hline$\sum$ PUFA & $1519.88 \pm 191.39$ & $1231.16 \pm 228.83$ & $1505.07 \pm 261.64$ & 0.8519 \\
\hline PUFA/SFA & $0.77 \pm 0.06$ & $0.68 \pm 0.06$ & $0.70 \pm 0.07$ & 0.0365 \\
\hline$\sum n-3 P U F A$ & $682.48 \pm 170.17$ & $356.63 \pm 72.64$ & $399.37 \pm 68.16$ & 0.0004 \\
\hline$\sum$ n-6PUFA & $1045.88 \pm 117.45$ & $1167.97 \pm 233.56$ & $1452.52 \pm 275.64$ & 0.0008 \\
\hline$n-6 / n-3 P U F A$ & $1.69 \pm 0.83$ & $3.30 \pm 0.43$ & $3.66 \pm 0.49$ & 0.0000 \\
\hline
\end{tabular}

" Abbreviations as in Table 2.

\subsection{Fatty Acid Profile of the Kidney}

The fatty acid profile of the kidney is presented in Table 5. Within the n-3 LC-PUFA, the contents of ALA, EPA, DHA, DPA, EPA + DHA, and EPA + DHA + DPA were greater in the omega-3 oil diet than in the control and MSM whole grain diets. The overall increase in the contents of 18:3 n-3 and its long chain metabolites remained statistically significant. A boxplot of Hochberg's adjusted multiple comparisons of significant differences between the treatment groups in kidney fatty acid profiles are depicted in Figure 3, where the omega-3 oil diet maintained a significantly higher fatty acid content than the control and MSM whole grain diets. However, the control diet had the highest contents of C22:0, C20:2n-6, 
C22:5n-6, C22:4n-6, and n-6/n-3 PUFA ratio, while the MSM whole grain diet led in C20:0 and $\mathrm{C} 21: 5 \mathrm{n}-3$ contents.

Table 5. Fatty acid profile $(\mathrm{mg} / 100 \mathrm{~g})$ of the kidney in TAW lambs ${ }^{\text {. }}$

\begin{tabular}{|c|c|c|c|c|}
\hline Fatty Acid & Omega-3 & Control & MSM Whole Grain & $p$-Value \\
\hline 13:0 & $0.22 \pm 0.21$ & $0.16 \pm 0.22$ & $0.28 \pm 0.22$ & 0.5721 \\
\hline $14: 1$ & $0.1 \pm 0.14$ & $0.05 \pm 0.15$ & $0.04 \pm 0.07$ & 0.2296 \\
\hline 14:0 & $7 \pm 2.29$ & $6.96 \pm 2.3$ & $5.42 \pm 1.27$ & 0.0886 \\
\hline $15: 1$ & $5.33 \pm 1.39$ & $5.73 \pm 1.45$ & $3.72 \pm 1.33$ & 0.0217 \\
\hline $16: 0$ & $317.14 \pm 52.86$ & $339.32 \pm 63.53$ & $283.75 \pm 48.67$ & 0.2055 \\
\hline $16: 1$ & $10.57 \pm 2.87$ & $11.21 \pm 2.56$ & $11.39 \pm 2.94$ & 0.5105 \\
\hline $17: 1 \mathrm{n} 8 \mathrm{c}+\mathrm{a} 17: 0$ & $12.44 \pm 1.91$ & $14.29 \pm 3.33$ & $12.04 \pm 2.77$ & 0.7594 \\
\hline $17: 0$ & $25.4 \pm 3.66$ & $28.7 \pm 7.39$ & $23.32 \pm 5.09$ & 0.4353 \\
\hline $18: 3 n 6$ & $1.75 \pm 0.54$ & $1.28 \pm 0.28$ & $0.98 \pm 0.25$ & 0.0001 \\
\hline $18: 4 n-3$ & $0.00 \pm 0.00$ & $0.04 \pm 0.12$ & $0.01 \pm 0.03$ & 0.7995 \\
\hline 18:2n-6 (LA) & $281.42 \pm 66.94$ & $280.63 \pm 73.32$ & $249.57 \pm 56.92$ & 0.2849 \\
\hline 18:3n-3 (ALA) & $12.43 \pm 3.02$ & $8.4 \pm 1.62$ & $7.63 \pm 1.61$ & 0.0001 \\
\hline CLA & $3.5 \pm 0.82$ & $3.78 \pm 0.85$ & $3.11 \pm 1.14$ & 0.3653 \\
\hline $18: 0$ & $351.69 \pm 51.59$ & $327.73 \pm 62.89$ & $279.14 \pm 47.83$ & 0.0054 \\
\hline $18: 1$ & $320.27 \pm 53.92$ & $314.51 \pm 56.7$ & $293.23 \pm 58.49$ & 0.2854 \\
\hline 19:1 & $2.17 \pm 0.44$ & $3.84 \pm 1.28$ & $2.9 \pm 1.01$ & 0.1645 \\
\hline 20:4n-6 (ARA) & $169.81 \pm 27.05$ & $246.86 \pm 57.97$ & $209.05 \pm 37.93$ & 0.0940 \\
\hline 20:5n-3 (EPA) & $69.1 \pm 17.67$ & $16.87 \pm 4.66$ & $16.57 \pm 2.71$ & 0.0000 \\
\hline $20: 3$ & $1.1 \pm 0.62$ & $2.98 \pm 0.75$ & $3.06 \pm 1.12$ & 0.0001 \\
\hline $20: 3 n-6$ & $19.6 \pm 3.26$ & $17.41 \pm 5.3$ & $12.88 \pm 3.59$ & 0.0010 \\
\hline $20: 4 n-3$ & $1.95 \pm 0.45$ & $1.91 \pm 0.92$ & $2.54 \pm 1.71$ & 0.2627 \\
\hline $20: 2 n-6$ & $7.9 \pm 1.65$ & $8.77 \pm 2.68$ & $6.62 \pm 2.3$ & 0.2320 \\
\hline 20:0 & $5.35 \pm 1.12$ & $5.84 \pm 1.13$ & $4.91 \pm 0.85$ & 0.3655 \\
\hline $20: 1$ & $11.88 \pm 2.14$ & $9.14 \pm 2.2$ & $8.52 \pm 2.19$ & 0.0020 \\
\hline $21: 5 n-3$ & $0.48 \pm 0.13$ & $0.99 \pm 0.38$ & $1.04 \pm 0.35$ & 0.0006 \\
\hline 21:0 & $0.69 \pm 0.13$ & $0.82 \pm 0.16$ & $0.65 \pm 0.13$ & 0.5464 \\
\hline $22: 5 n-6$ & $0.44 \pm 0.49$ & $3.03 \pm 0.73$ & $2.33 \pm 0.62$ & 0.0003 \\
\hline 22:6n-3 (DHA) & $58.76 \pm 10.76$ & $25.17 \pm 5.59$ & $25.3 \pm 5.21$ & 0.0000 \\
\hline 22:4n-6 & $3.52 \pm 0.76$ & $15.04 \pm 5.17$ & $9.88 \pm 3.32$ & 0.0133 \\
\hline 22:5n-3 (DPA) & $43.39 \pm 6.14$ & $33.76 \pm 8.04$ & $29.14 \pm 4.96$ & 0.0000 \\
\hline 22:0 & $35.02 \pm 6.6$ & $36.52 \pm 8.02$ & $29.06 \pm 5.3$ & 0.0000 \\
\hline 22:1 & $9.01 \pm 1.71$ & $5.12 \pm 2.16$ & $3.62 \pm 1.08$ & 0.0659 \\
\hline $23: 1$ & $0.56 \pm 0.23$ & $0.96 \pm 0.34$ & $0.8 \pm 0.28$ & 0.1012 \\
\hline 23:0 & $8.84 \pm 1.35$ & $9.96 \pm 1.97$ & $7.94 \pm 1.71$ & 0.2826 \\
\hline 24:1 & $30.55 \pm 5.54$ & $33.12 \pm 7.47$ & $32.73 \pm 7.26$ & 0.4763 \\
\hline 24:0 & $34.42 \pm 5.47$ & $37.53 \pm 7.62$ & $31.21 \pm 6.79$ & 0.3120 \\
\hline Total FA & $1499.2 \pm 208.97$ & $1515.42 \pm 308.98$ & $1322.14 \pm 193.73$ & 0.1155 \\
\hline $\mathrm{EPA}+\mathrm{DHA}$ & $127.86 \pm 26.2$ & $42.04 \pm 9.14$ & $41.87 \pm 7.28$ & 0.0000 \\
\hline $\mathrm{EPA}+\mathrm{DHA}+\mathrm{DPA}$ & $171.26 \pm 29.76$ & $75.8 \pm 15.82$ & $71.01 \pm 10.98$ & 0.0000 \\
\hline$\sum$ SFA & $439.41 \pm 67.09$ & $471.55 \pm 89.38$ & $390.25 \pm 66.92$ & 0.1743 \\
\hline$\sum$ MUFA & $385.11 \pm 61.94$ & $377.95 \pm 69.57$ & $353.22 \pm 71.1$ & 0.2933 \\
\hline$\sum$ PUFA & $674.69 \pm 91.83$ & $665.92 \pm 156.17$ & $578.66 \pm 79.86$ & 0.0698 \\
\hline PUFA/SFA & $1.54 \pm 0.13$ & $1.4 \pm 0.12$ & $1.51 \pm 0.29$ & 0.7367 \\
\hline$\sum n-3 P U F A$ & $187.22 \pm 32.15$ & $90.12 \pm 18.16$ & $85.28 \pm 13.7$ & 0.0000 \\
\hline$\sum$ n-6PUFA & $318.13 \pm 70.45$ & $329.97 \pm 86.61$ & $285.38 \pm 60.57$ & 0.3264 \\
\hline n-6/n-3PUFA & $1.74 \pm 0.42$ & $3.65 \pm 0.64$ & $3.44 \pm 1.14$ & 0.0003 \\
\hline
\end{tabular}

\footnotetext{
\# Abbreviations as in Table 2.
}

\subsection{Fatty Acid Profile of the Heart}

Table 6 shows the fatty acid contents of the heart. The hearts from lambs on the omega-3 oil diet had the highest ALA, EPA, C20:2n-6, EPA + DHA, EPA + DHA + DPA, n-3 LC-PUFA, DHA, and DPA contents and lowest $n-6 / n-3$ PUFA ratio than in the control and MSM whole grain diets. However, the hearts of lambs fed the control diet had the highest contents of C23:0, C22:0m and C22:4n-6, while those on the MSM whole grain diet had 
Kidney
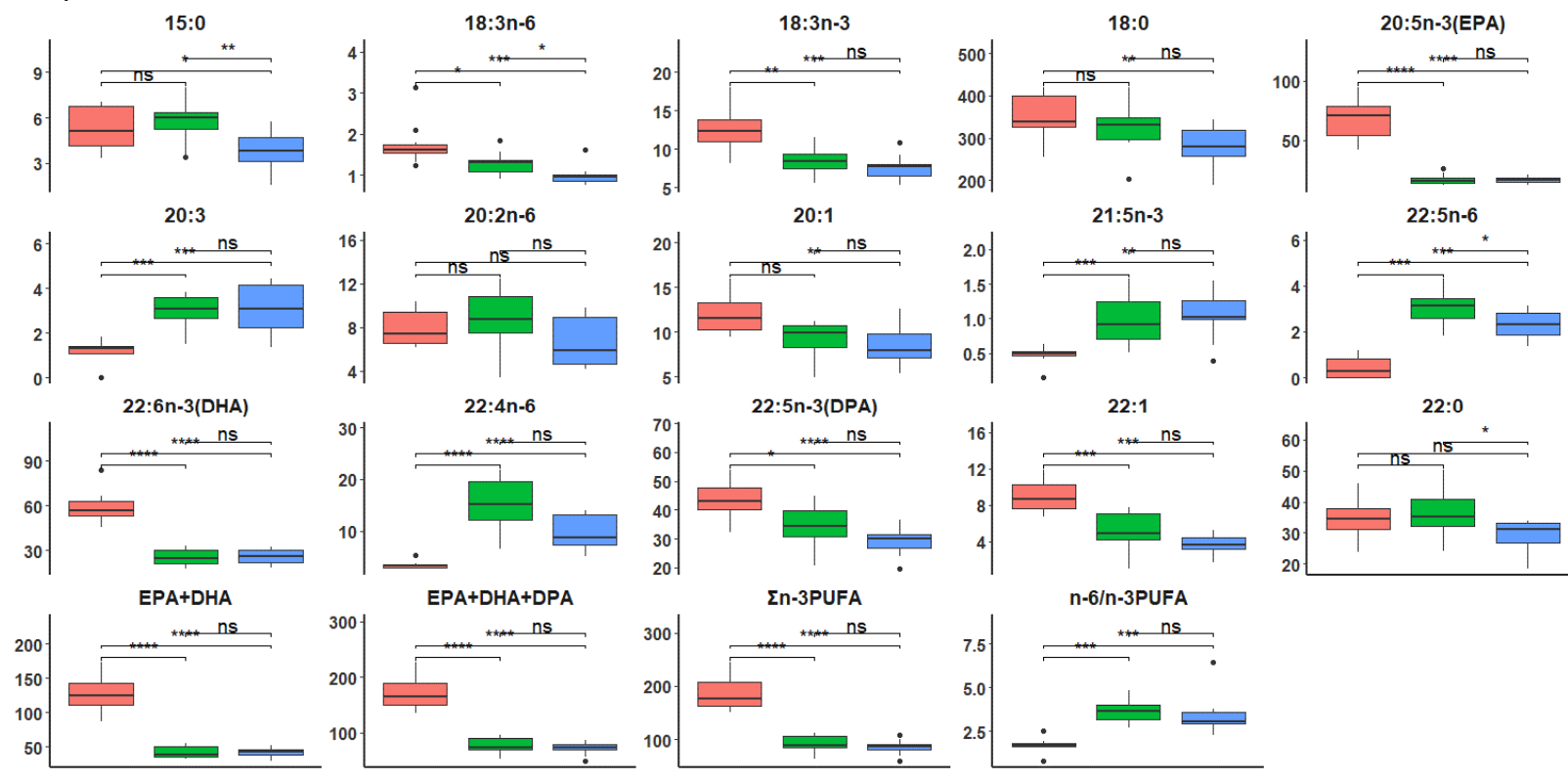

宁 Omega Control

Figure 3. Boxplots showing the distribution of selected fatty acids composition in the kidney. Each plot tested the mean fatty acid in omega-3 versus control, omega-3 versus MSM whole grain and control versus MSM whole grain with Hochberg's adjusted multiple comparisons. ${ }^{*} p<0.05{ }^{* *} p<0.01 ;{ }^{* * *} p<0.001 ;{ }^{* * * *} p<0.0001$; ns, not significant $(p>0.05)$.
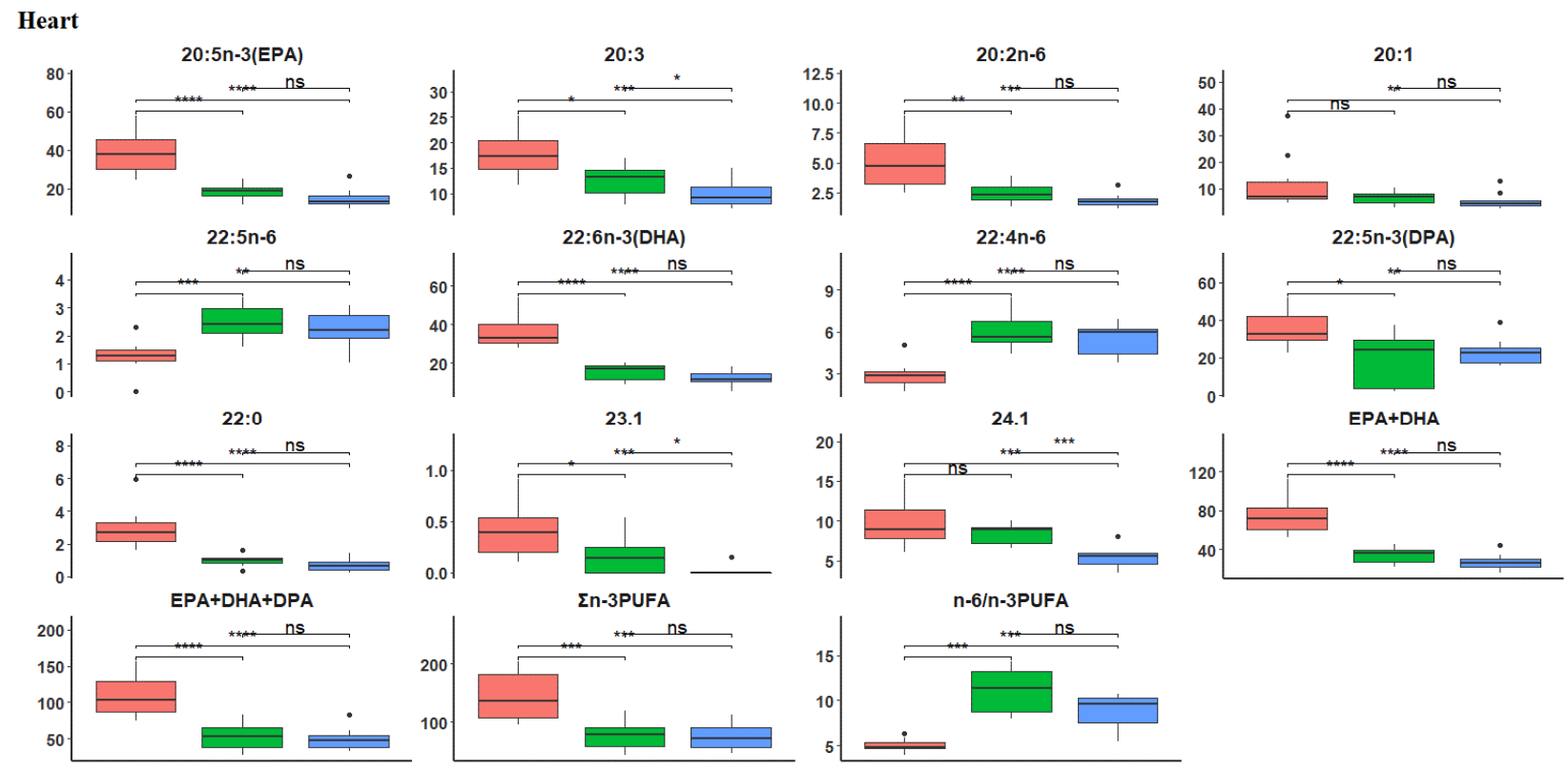

Omega Control

Figure 4. Boxplots showing the distribution of selected fatty acids composition in the heart. Each plot tested the mean fatty acid in omega-3 versus control, omega-3 versus MSM whole grain, and control versus MSM whole grain with Hochberg's adjusted multiple comparisons. ${ }^{*} p<0.05 ;{ }^{* *} p<0.01 ;{ }^{* * *} p<0.001 ;{ }^{* * * *} p<0.0001$; ns, not significant $(p>0.05)$. 
Table 6. Fatty acid profile (mg/100 g) of the heart in TAW lambs \#.

\begin{tabular}{|c|c|c|c|c|}
\hline Fatty Acid & Omega-3 & Control & MSM Whole Grain & $p$-Value \\
\hline $13: 0$ & $0.14 \pm 0.35$ & $0.02 \pm 0.07$ & $0.03 \pm 0.1$ & 0.2533 \\
\hline $14: 1$ & $0.51 \pm 0.35$ & $0.61 \pm 0.3$ & $0.36 \pm 0.47$ & 0.3935 \\
\hline $14: 0$ & $45.97 \pm 95.07$ & $13.63 \pm 9.65$ & $23.69 \pm 32.81$ & 0.3994 \\
\hline $15: 1$ & $11.42 \pm 20.53$ & $4.75 \pm 2.84$ & $6.68 \pm 5.79$ & 0.3391 \\
\hline $16: 0$ & $539.22 \pm 573.68$ & $402.04 \pm 132.98$ & $389.7 \pm 233.75$ & 0.3616 \\
\hline $16: 1$ & $59.06 \pm 91.19$ & $34.61 \pm 18.23$ & $41.34 \pm 35.23$ & 0.4919 \\
\hline $17: 1 \mathrm{n} 8 \mathrm{c}+\mathrm{a} 17: 0$ & $33.28 \pm 40.65$ & $21.73 \pm 10.42$ & $26.43 \pm 18.68$ & 0.5655 \\
\hline $17: 0$ & $57.87 \pm 82.96$ & $37.03 \pm 17.28$ & $41.53 \pm 29.75$ & 0.4822 \\
\hline $18: 3 n 6$ & $2.81 \pm 1.01$ & $2.55 \pm 0.55$ & $2.24 \pm 0.57$ & 0.0919 \\
\hline $18: 4 n-3$ & $0.31 \pm 0.46$ & $0.15 \pm 0.29$ & $0.06 \pm 0.12$ & 0.0823 \\
\hline $18: 2 n-6(L A)$ & $543.33 \pm 141.44$ & $591.85 \pm 100.17$ & $466.72 \pm 122.48$ & 0.1901 \\
\hline 18:3n-3 (ALA) & $31.32 \pm 26.24$ & $18.55 \pm 7.28$ & $22.03 \pm 15.47$ & 0.2636 \\
\hline CLA & $709.39 \pm 866.87$ & $492.75 \pm 233.75$ & $459.86 \pm 332.73$ & 0.3144 \\
\hline 18:0 & $862.44 \pm 1163.49$ & $557.84 \pm 334.03$ & $601.47 \pm 462.38$ & 0.4367 \\
\hline $18: 1$ & $9.67 \pm 10.06$ & $6.03 \pm 2.24$ & $5.68 \pm 3$ & 0.1578 \\
\hline $19: 1$ & $3.32 \pm 3.49$ & $2.59 \pm 1.28$ & $3.41 \pm 2.54$ & 0.9383 \\
\hline $20: 4 n-6$ (ARA) & $128.74 \pm 35.44$ & $166.3 \pm 33.44$ & $143.74 \pm 48.1$ & 0.4257 \\
\hline 20:5n-3 (EPA) & $38.59 \pm 11.44$ & $18.01 \pm 3.9$ & $14.87 \pm 4.96$ & 0.0000 \\
\hline $22: 3$ & $1.6 \pm 0.83$ & $3.05 \pm 0.79$ & $2.23 \pm 0.62$ & 0.1398 \\
\hline $20: 3 n-6$ & $18.01 \pm 4.67$ & $12.78 \pm 3.01$ & $9.71 \pm 2.57$ & 0.0000 \\
\hline $20: 4 n-3$ & $0.22 \pm 0.28$ & $0.48 \pm 0.72$ & $0.11 \pm 0.24$ & 0.6342 \\
\hline $20: 2 n-6$ & $5.12 \pm 2.19$ & $2.45 \pm 0.87$ & $1.82 \pm 0.6$ & 0.0000 \\
\hline 20:0 & $6.11 \pm 8.08$ & $4.76 \pm 2.14$ & $4.27 \pm 2.66$ & 0.4158 \\
\hline 20:1 & $11.82 \pm 10.49$ & $6.45 \pm 2.36$ & $5.24 \pm 3.19$ & 0.0301 \\
\hline $21: 5 n-3$ & $0.29 \pm 0.55$ & $0.62 \pm 0.32$ & $0.39 \pm 0.26$ & 0.6112 \\
\hline 21:0 & $0.62 \pm 0.74$ & $0.48 \pm 0.13$ & $0.61 \pm 0.46$ & 0.9544 \\
\hline $22: 5 n-6$ & $1.26 \pm 0.58$ & $2.49 \pm 0.57$ & $2.21 \pm 0.64$ & 0.0045 \\
\hline 22:6n-3 (DHA) & $36.74 \pm 9.42$ & $15.37 \pm 4.1$ & $11.99 \pm 3.73$ & 0.0000 \\
\hline $22: 4 n-6$ & $2.91 \pm 0.89$ & $6.06 \pm 1.29$ & $5.5 \pm 1.16$ & 0.0003 \\
\hline $22: 5 n-3$ (DPA) & $34.82 \pm 9.38$ & $19.03 \pm 14.44$ & $23 \pm 6.92$ & 0.0301 \\
\hline $22: 0$ & $2.95 \pm 1.25$ & $0.97 \pm 0.33$ & $0.69 \pm 0.38$ & 0.0000 \\
\hline $22: 1$ & $4.47 \pm 2.07$ & $5.03 \pm 1.97$ & $3.72 \pm 1.83$ & 0.4011 \\
\hline $23: 1$ & $0.43 \pm 0.28$ & $0.17 \pm 0.19$ & $0.02 \pm 0.05$ & 0.0000 \\
\hline $23: 0$ & $4.58 \pm 1.46$ & $6.38 \pm 1.24$ & $4.43 \pm 1.42$ & 0.8409 \\
\hline $24: 1$ & $10.03 \pm 3.11$ & $8.43 \pm 1.31$ & $5.59 \pm 1.55$ & 0.0000 \\
\hline 24:0 & $4.19 \pm 1.24$ & $4.65 \pm 1.82$ & $3.72 \pm 1.03$ & 0.4712 \\
\hline Total FA & $3223.54 \pm 3074.77$ & $2470.68 \pm 880$ & $2335.08 \pm 1176.42$ & 0.3140 \\
\hline $\mathrm{EPA}+\mathrm{DHA}$ & $75.32 \pm 20.32$ & $33.38 \pm 7.73$ & $26.86 \pm 8.05$ & 0.0000 \\
\hline $\mathrm{EPA}+\mathrm{DHA}+\mathrm{DPA}$ & $110.14 \pm 28.19$ & $52.41 \pm 18.48$ & $49.86 \pm 14.91$ & 0.0000 \\
\hline$\sum \mathrm{SFA}$ & $1384 \pm 1645.79$ & $971.52 \pm 396.97$ & $938.25 \pm 632.49$ & 0.3409 \\
\hline$\sum M U F A$ & $983.83 \pm 1309.31$ & $633.39 \pm 366.3$ & $684.53 \pm 519.99$ & 0.4274 \\
\hline$\sum$ PUFA & $855.71 \pm 225.88$ & $865.77 \pm 149$ & $712.3 \pm 183.06$ & 0.1016 \\
\hline PUFA/SFA & $1 \pm 0.42$ & $0.98 \pm 0.25$ & $0.99 \pm 0.48$ & 0.9636 \\
\hline$\sum n-3 P U F A$ & $143.88 \pm 41.03$ & $75.26 \pm 23.68$ & $74.68 \pm 23.06$ & 0.0000 \\
\hline$\sum$ n-6PUFA & $711.84 \pm 189.55$ & $790.5 \pm 130.94$ & $637.62 \pm 168.09$ & 0.3408 \\
\hline$n-6 / n-3 P U F A$ & $5.01 \pm 0.68$ & $11.12 \pm 2.43$ & $8.82 \pm 1.92$ & 0.0041 \\
\hline
\end{tabular}

\# Abbreviations as in Table 2.

\section{Discussion}

A prospective cohort study of men in the USA [45] and a cross-sectional survey of Korean adults [46] both reinforced the need to enhance a healthier composition of red meat among consumers to minimize the dietary risks of coronary heart disease, cardiometabolic, and cancer mortality burdens. Previous studies [22,47-51] had established that the fatty acid profiles of muscles and organs can be modified by dietary supplementation with $n-3$ LC-PUFA, leading to higher human health beneficial EPA + DHA + DPA and lower n-3/n-6 ratio [52]. It is important to strike a balance between attaining higher total PUFA deposition in the muscle and oxidative stability [53] because meat color, flavor, nutritional value, 
shelf-life, and overall consumer acceptance can be compromised by lipid oxidation [54,55]. Therefore, in this study, the time-tested and oxidatively stable omega-3 oil infused pellets previously reported in Le et al. [49] were utilized in the comparative analysis with non-oil and whole grain pellets.

The level of incorporation and abundance of both monounsaturated (MUFA) and polyunsaturated (PUFA) fatty acids in the muscle is consistent with previously reported intramuscular fatty acid compositions [56,57]. A review of pre-clinical and human trials with conjugated linoleic acid CLA (C18:2n-6) revealed positive effects on cancer, obesity, and atherosclerosis $[58,59]$, and the muscle in the present study had significantly incorporated levels of CLA and ALA (C18:3n-3). ALA is the precursor for the synthesis of n-3 LC-PUFA through desaturation and/or chain-elongation by desaturase and elongase enzymes $[60,61]$. In the present study, it was evident that supplementation of lambs with omega-3 oil-fortified pellets increased the muscle contents of ALA by two-to three-folds, which translated into higher contents of EPA + DHA + DPA than in muscles from the control and whole grain diets. Furthermore, the high and significant levels of C20:4n-6 in the muscle could be a result of LA being subjected to elongation and desaturation by delta- 5 and delta- 6 desaturases and elongase enzymes [62], since all dietary treatments favored de novo fatty acid synthesis due to high levels of C18:1 originating from elongation and desaturation of C16:0 into palmitoleic and C18:0 into oleic acids [63-65]. In terms of dietary n-6/n-3 PUFA ratio, the human nutrition guidelines recommend an ideal value of not more than 4 [66], because n-3 PUFA plays an anti-inflammatory role [67], while n-6 PUFA exerts pro-inflammatory effects [68] in asthma and rheumatoid arthritis [69] and increased risk of cancer [70]. This study clearly demonstrated that supplementing lambs with omega- 3 oil is an excellent nutritional strategy for lowering the $n-6 / n-3$ ratio in the muscle for a healthier meat.

The liver is responsible for the metabolism, uptake, and dissemination of lipids through free fatty acids, lipoproteins, and de novo lipogenesis [71]. It plays a role in mitochondrial fatty acid $\beta$-oxidation and facilitates key catabolic pathways in hepatocytes [71] and ruminant meat production [72]. In the liver of lambs supplemented with omega-3 oil, the significantly higher proportions of EPA + DHA + DPA compared to the control diet equivalent to three-folds of what is obtainable in the kidney and heart (Tables 5 and 6 ) in the present study, align with earlier reports [35,49,51]. These figures are well above the Food Standards of Australia and New Zealand recommendations of $30 \mathrm{mg} / 100 \mathrm{~g}$ as 'source' and $60 \mathrm{mg} / 100 \mathrm{~g}$ as 'good source' levels [49], and are in agreement with previous studies showing that the lamb liver is one of the richest sources of EPA + DHA + DPA, comprising approximately $185 \mathrm{mg} / 100 \mathrm{~g}$ of hepatocytes from omega-3 oil supplemented diet [73]. The liver levels of CLA were also high in lambs supplemented with omega-3 oil, and given its reported immuno-modulatory, anti-obesity, and anti-carcinogenic properties [74,75], the liver could be considered a healthy product. The major synthesis pathway of the CLA isomer, rumenic acid, in the tissues of ruminants is primarily from the biohydrogenation of ALA into vaccenic acid (C18:1n-7) catalyzed by reductase enzymes [76,77]. The variation patterns and ranges in liver fatty acid profiles in the present study were similar to previously reported findings [78-83]. The liver has also been reported to have a high nutrient content of essential amino acids, fatty acids, iron, zinc, magnesium, selenium, calcium, vitamins B1, B6, B12, and folic acid $[84,85]$ and may explain why duck liver attracts a price premium and is consumed in France [86].

Mashek and Coleman [87] showed that the kidney plays a role in cellular fatty acid uptake and contributes to metabolism, with strong suggestions that the metabolic demand for fatty acids is a major driving force governing fatty acid uptake in the kidney. Although the mechanism is unknown, it appears that converting fatty acids to acyl-CoAs and downstream metabolic intermediates increases cellular fatty acid uptake, probably by limiting efflux [87]. Hagve et al. [88] demonstrated that increasing levels of $n-3$ fatty acids in membranes affect the uptake and intracellular metabolism of fatty acids as well as membrane fluidity in the kidney. Evidence from both gain and loss-of-function experiments indicate 
that fatty acid uptake can be modulated by activation at both the plasma membrane and internal organ sites by intracellular fatty acid binding proteins, and by enzymes in synthetic or degradative metabolic pathways [87]. In a study of long-chain polyunsaturated fatty acids metabolism in kidney cells, Liabo et al. [89] argued that only little is known about the metabolism of fatty acids in the kidney, because it is controversial whether the kidney possesses the ability to desaturate long-chain fatty acids or kidney cells are dependent on pre-formed polyunsaturated fatty acids transported from the liver. However, they concluded that the kidney, at least in part, must obtain its C-20 and C-22 fatty acids from circulation, while the active delta5-desaturase suggests that pre-formed C-20 fatty acids can be converted to more unsaturated homologues in the kidney. This could probably explain the significant increases in the contents of C18:3n-3 and its long chain ALA, EPA, DHA, and DPA metabolites being greater in the kidney of lambs supplemented with omega-3 oil than in the control and MSM whole grain diets observed in the present study (Table 4 and Figure 3).

Schaap et al. [90] reported that long-chain fatty acids are important fuel molecules in the heart, because their oxidation in the mitochondria provides the bulk of the energy required for cardiac functioning. However, the cellular transport of fatty acids in aqueous solutions is impaired due to low solubility. To circumvent this hurdle, cardiac tissues contain several fatty acid-binding proteins (FABP) capable of non-covalently binding to fatty acids, thus facilitating both cellular uptake and intracellular transport of fatty acids. The majority of fatty acids taken up by the heart seems to pass the sarcolemma through a carrier-mediated translocation mechanism consisting of one or more membrane-associated FABP [81]. Perhaps the observed significant differences between the treatment groups in the heart fatty acid profiles in the present study where the omega- 3 oil diet maintained a significantly higher EPA, DHA, EPA + DHA, EPA + DHA + DPA, and En-3PUFA content than the control and MSM whole grain diets, could probably be indicative of higher activities of the FABP in intracellular transport and cellular uptake of long chain fatty acids.

\section{Conclusions}

This is the first study that evaluated and compared the fatty acid profiles in the tissues and organs of TAW MARGRA lambs raised in a feedlot production system in response to dietary supplementation with or without fortification with omega-3 oil. It was primarily to shed some light on n-3 LC-PUFA metabolism in the Longissimus thoracis et lumborum muscle, heart, kidney, and liver of lot-fed TAW MARGRA lambs in response to dietary supplementation with omega-3 oil. The findings suggest that dietary manipulation can be utilized to improve the fatty acid content and nutritional value of muscle and organs of TAW MARGRA lambs to meat that more closely meets nutritional guidelines of higher levels of health-beneficial n-3 LC-PUFA. The data clearly portray the liver, kidney, and heart of TAW MARGRA lambs with highest contents of the healthiest omega-3 fatty acids well beyond the FSANZ 'good source' levels. Therefore, the hypothesis that fortifying feedlot pellets with omega-3 oil will enhance the human health beneficial n-3 LC-PUFA composition of edible lamb muscle tissue and organs holds, and is worthy of acceptance.

Author Contributions: Conceptualization, A.E.O.M.-A.; Methodology, A.E.O.M.-A., J.R.O. and S.B.P., Software, A.E.O.M.-A.; Validation, A.E.O.M.-A., S.B.P. and J.R.O., Formal analysis, S.B.P. and O.A.A.; Investigation, S.B.P.; Resources, A.E.O.M.-A., R.T.K. and O.A.A.; Data curation, writing-original draft preparation, S.B.P.; Writing-reviewing and editing, A.E.O.M.-A., R.T.K. and O.A.A.; Supervision, A.E.O.M.-A., R.T.K. and O.A.A.; Project administration, A.E.O.M.-A.; Funding acquisition, A.E.O.M.-A. All authors have read and agreed to the published version of the manuscript.

Funding: This research was funded by the Innovation Connections Research Grant from the Australian Commonwealth Government's Department of Industry, Science, Energy and Resources, Science Industry Endowment Fund Ross Metcalf STEM Business Fellowship (awarded to J.R.O.), Tattykeel Australian White Pty Ltd., and a PhD scholarship funded by the James Cook University Postgraduate Research Scholarship (JCUPRS), Queensland, Australia, awarded to the first named author (S.B.P.). 
Institutional Review Board Statement: The James Cook University Animal Ethics Committee (Permit No. A0015657) approved the use of animals and all procedures executed in this study in compliance with the Australian Code for Care and Use of Animals for Scientific Purposes (Eighth edition, 2013).

Informed Consent Statement: Not applicable.

Data Availability Statement: Data available upon reasonable request from authors.

Acknowledgments: The authors gratefully acknowledge JCUPRS, James Cook University College of Public Health, Medical and Veterinary Sciences (PhD scholarship for the first-named authorS.B.P.), the Australian Commonwealth Department of Industry, Science and Resources Innovation Connections, the Commonwealth Scientific and Industrial Research Organization SIEF Ross Metcalf STEM Business Industrial Research Fellowship (research funding for the second-named authorJ.R.O.), Tattykeel Australian White Pty Ltd. (access to flock, farm resources, research funding), CSIRO Marine and Atmosphere Hobart (fatty acid analysis), and the National Veterinary Research Institute Vom, Nigeria (study leave approval for the first-named author-S.B.P.).

Conflicts of Interest: The authors declare no conflict of interest. The funders had no role in the study's design of the study, collection, analyses, or interpretation of data, in the writing of the manuscript, or in the decision to publish the results.

\section{References}

1. Boncinelli, F.; Piracci, G.; Casini, L. Understanding the role of information and taste heterogeneity in consumer preferences for functional beef: The case of the omega-3 enriched burger. Meat Sci. 2021, 181, 108614. [CrossRef]

2. Munekata, P.E.S.; Perez-Alvarez, J.A.; Pateiro, M.; Viuda-Matos, M.; Fernandez-Lopez, J.; Lorenzo, J.M. Satiety from healthier and functional foods. Trends Food Sci. Technol. 2021, 113, 397-410. [CrossRef]

3. Ansorena, D.; Astiasarán, I. Enrichment of meat products with omega-3 fatty acids by methods other than modification of animal diet. In Food Enrichment with Omega-3 Fatty Acids; Woodhead Publishing Series in Food Science, Technology and Nutrition; Woodhead Publishing: Sawston, UK, 2013; Chapter 10; pp. 299-318.

4. Morsy, M.K.; Elsabagh, R. Quality parameters and oxidative stability of functional beef burgers fortified with microencapsulated cod liver oil. LWT Food Sci. Technol. 2021, 142, 110959. [CrossRef]

5. Barros, J.C.; Munekata, P.E.S.; Carvalho, F.A.L.; Dominguez, R.; Trindade, M.A.; Pateiro, M.; Lorenzo, J.M. Healthy beef burgers: Effect of animal fat replacement by algal and wheat germ oil emulsions. Meat Sci. 2021, 173, 108396. [CrossRef]

6. Van Vliet, S.; Kronberg, S.L.; Provenza, F.D. Plant-based meats, human health, and climate change. Front. Sustain. Food Syst. 2020, 4, 128. [CrossRef]

7. Bohrer, B.M. Nutrient density and nutritional value of meat products and non-meat foods high in protein. Trends Food Sci. Technol. 2017, 65, 103-112. [CrossRef]

8. Juárez, M.; Lam, S.; Bohrer, B.M.; Dugan, M.E.; Vahmani, P.; Aalhus, J.; Juárez, A.; López-Campos, O.; Prieto, N.; Segura, J. Enhancing the nutritional value of red meat through genetic and feeding strategies. Foods 2021, 10, 872. [CrossRef]

9. Fu, Y.; Wang, Y.; Gao, H.; Li, D.; Jiang, R.; Ge, L.; Tong, C.; Xu, K. Associations among dietary omega-3 polyunsaturated fatty acids, the gut microbiota, and intestinal immunity. Mediat. Inflamm. 2021, 2021, 8879227. [CrossRef]

10. Ponnampalam, E.N.; Sinclair, A.J.; Holman, B.W. The sources, synthesis and biological actions of omega-3 and omega-6 fatty acids in red meat: An overview. Foods 2021, 10, 1358. [CrossRef]

11. Rizos, E.C.; Markozannes, G.; Tsapas, A.; Mantzoros, C.S.; Ntzani, E.E. Omega-3 supplementation and cardiovascular disease: Formulation-based systematic review and meta-analysis with trial sequential analysis. Heart 2021, 107, 150-158. [CrossRef]

12. Chappus-McCendie, H.; Chevalier, L.; Roberge, C.; Plourde, M. Omega-3 PUFA metabolism and brain modifications during aging. Prog. Neuropsychopharmacol. Biol. Psychiatry 2019, 94, 109662. [CrossRef]

13. Suito, T.; Nagao, K.; Takeuchi, K.; Juni, N.; Hara, Y.; Umeda, M. Functional expression of $\Delta 12$ fatty acid desaturase modulates thermoregulatory behaviour in Drosophila. Sci. Rep. 2020, 10, 11798. [CrossRef]

14. Mazzocchi, A.; De Cosmi, V.; Risé, P.; Milani, G.P.; Turolo, S.; Syrén, M.-L.; Sala, A.; Agostoni, C. Bioactive compounds in edible oils and their role in oxidative stress and inflammation. Front. Physiol. 2021, 12, 659551. [CrossRef]

15. Núñez-Sánchez, N.; Avilés Ramírez, C.; Peña Blanco, F.; Gómez-Cortés, P.; de la Fuente, M.Á.; Vioque Amor, M.; Horcada Ibáñez, A.; Martínez Marín, A.L. Effects of algae meal supplementation in feedlot lambs with competent reticular groove reflex on growth performance, carcass traits and meat characteristics. Foods 2021, 10, 857. [CrossRef] [PubMed]

16. Sharafi, Y.; Majidi, M.M.; Goli, S.A.H.; Rashidi, F. Oil content and fatty acids composition in Brassica species. Int. J. Food Prop. 2015, 18, 2145-2154. [CrossRef]

17. Jokić, S.; Sudar, R.; Svilović, S.; Vidović, S.; Bilić, M.; Velić, D.; Jurković, V. Fatty acid composition of oil obtained from soybeans by extraction with supercritical carbon dioxide. Czech J. Food Sci. 2013, 31, 116-125. [CrossRef]

18. Giuffrè, A.M.; Capocasale, M. Physicochemical composition of tomato seed oil for an edible use: The effect of cultivar. Int. Food Res. J. 2016, 23, 583-591. 
19. Angelo, M.G.; Nobile, R. Citrus bergamia, Risso: The peel, the juice and the seed oil of the bergamot fruit of Reggio Calabria (South Italy). Emir. J. Food Agric. 2020, 32, 522-532.

20. Gonzales-Barron, U.; Popova, T.; Piedra, R.B.; Tolsdorf, A.; Geß, A.; Pires, J.; Domínguez, R.; Chiesa, F.; Brugiapaglia, A.; Viola, I.; et al. Fatty acid composition of lamb meat from Italian and German local breeds. Small Rum. Res. 2021, 200, 106384. [CrossRef]

21. Hoffman, L.C.; Claasen, B.; der Merwe, V.; André, D.; Cloete, S.W.P.; Cloete, J.J.E. The effects of production system and sex on the sensory quality characteristics of Dorper lamb. Foods 2020, 9, 725. [CrossRef]

22. Malau-Aduli, A.E.O.; Nguyen, D.V.; Le, H.V.; Nguyen, Q.V.; Otto, J.R.; Malau-Aduli, B.S.; Nichols, P.D. Correlations between growth and wool quality traits of genetically divergent Australian lambs in response to canola or flaxseed oil supplementation. PLoS ONE 2019, 14, e0208229. [CrossRef] [PubMed]

23. Bhuiyan, M.; Lee, D.; Kim, H.; Lee, S.; Cho, S.; Yang, B.; Kim, S.D.; Lee, S.H. Estimates of genetic parameters for fatty acid compositions in the longissimus dorsi muscle of Hanwoo cattle. Animal 2018, 12, 675-683. [CrossRef] [PubMed]

24. Junior, F.M.V.; Martins, C.F.; Feijó, G.L.D.; Teixeira, A.; Leonardo, A.P.; de Almeida Ricardo, H.; Fernandez, A.R.M.; Reis, F.A Evaluation of genotype on fatty acid profile and sensory of meat of indigenous Pantaneiro sheep and Texel or Santa Inês crossbred finished on feedlot. Small Rum. Res. 2019, 173, 17-22. [CrossRef]

25. Sarı, M.; Aksoy, Y.; Önk, K.; Erinç, H.; Işık, S.A.; Tilki, M. Effects of genotype and fattening system on the quality of male lamb meat-Part 1: Technological properties and carcass measurements. Arch. Anim. Breed 2019, 62, 605-614. [CrossRef]

26. Belaunzaran, X.; Lavín, P.; Mantecón, A.; Kramer, J.; Aldai, N. Effect of slaughter age and feeding system on the neutral and polar lipid composition of horse meat. Animal 2018, 12, 417-425. [CrossRef]

27. Miguel, E.; Blázquez, B.; Ruiz de Huidobro, F. Liveweight and sex effects on sensory quality of Rubia de El Molar autochthonous ovine breed meat. Animals 2021, 11, 1293. [CrossRef]

28. Matar, A.M.; Abdelrahman, M.M.; Alhidary, I.A.; Ayadi, M.A.; Alobre, M.M.; Aljumaah, R.S. Effects of roughage quality and particle size on rumen parameters and fatty acid profiles of Longissimus dorsi fat of lambs fed complete feed. Animals 2020, 10, 2182. [CrossRef]

29. Wood, J.; Enser, M. Manipulating the fatty acid composition of meat to improve nutritional value and meat quality. In New Aspects of Meat Quality; Peter, P.P., Ed.; Woodhead Publishing Series in Food Science, Technology and Nutrition; Woodhead Publishing: Sawston, UK, 2017; Volume 501-535, 744p.

30. Vahmani, P.; Ponnampalam, E.N.; Kraft, J.; Mapiye, C.; Bermingham, E.N.; Watkins, P.J.; Proctor, S.D.; Dugan, M.E.R. Bioactivity and health effects of ruminant meat lipids. Invited Review. Meat Sci. 2020, 165, 108114. [CrossRef]

31. Garcia-Galicia, I.A.; Arras-Acosta, J.A.; Huerta-Jimenez, M.; Rentería-Monterrubio, A.L.; Loya-Olguin, J.L.; Carrillo-Lopez, L.M.; Tirado-Gallegos, J.M.; Alarcon-Rojo, A.D. Natural oregano essential oil may replace antibiotics in lamb diets: Effects on meat quality. Antibiotics 2020, 9, 248. [CrossRef]

32. Dewanckele, L.; Vlaeminck, B.; Hernandez-Sanabria, E.; Ruiz-González, A.; Debruyne, S.; Jeyanathan, J.; Veerle, F. Rumen biohydrogenation and microbial community changes upon early life supplementation of 22: 6n-3 enriched microalgae to goats. Front. Microbiol. 2018, 9, 573. [CrossRef]

33. Holman, B.W.B.; Kerr, M.J.; Refshauge, G.; Diffey, S.M.; Hayes, R.C.; Newell, M.T.; Hopkins, D.L. Post-mortem pH decline in lamb semitendinosus muscle and its relationship to the $\mathrm{pH}$ decline parameters of the longissimus lumborum muscle: A pilot study. Meat Sci. 2021, 176, 108473. [CrossRef]

34. AOAC International. Official Methods of Analysis of AOAC International, 16th ed.; AOAC International: Gaithersburg, MD, USA, 1995.

35. Malau-Aduli, A.E.O.; Holman, B.W.B.; Kashani, A.; Nichols, P.D. Sire breed and sex effects on the fatty acid composition and content of heart, kidney, liver, adipose and muscle tissues of purebred and first-cross prime lambs. Anim. Prod. Sci. 2016, 56, 2122-2132. [CrossRef]

36. Malau-Aduli, A.E.O.; Siebert, B.D.; Bottema, C.D.K.; Pitchford, W.S. Breed comparison of the fatty acid composition of muscle phospholipids in Jersey and Limousin cattle. J. Anim. Sci. 1998, 76, 766-773. [CrossRef]

37. Bligh, E.G.; Dyer, W.J. A rapid method of total lipid extraction and purification. Can. J. Biochem. Physiol. 1959, 37, 911-917. [CrossRef]

38. Miller, M.R.; Nichols, P.D.; Barnes, J.; Davies, N.W.; Peacock, E.J.; Carter, C.G. Regiospecificity profiles of storage and membrane lipids from the gill and muscle tissue of Atlantic salmon (Salmo salar L.) grown at elevated temperature. Lipids 2006, 41, 865-876. [CrossRef]

39. Clayton, E. Graham Centre Monograph no. 4: Long-Chain Omega-3 Polyunsaturated Fatty Acids in Ruminant Nutrition: Benefits to Animals and Humans; Nugent, T., Nicholls, C., Eds.; New South Wales Department of Primary Industries: Wagga Wagga, Australia, 2014; ISBN 9781742566788.

40. R Core Team. R: A Language and Environment for Statistical Computing, Rstudio version 1.3.1056; R Foundation for Statistical Computing: Vienna, Austria, 2021; ISBN 3-900051-07-0. Available online: http://www.R-project.org/ (accessed on 20 August 2021).

41. Pohlert, T. The pairwise multiple comparison of mean ranks package (PMCMR). R Package 2014, $27,9$.

42. Dunn, O.J. Multiple comparisons using rank sums. Technometrics 1964, 6, 241-252. [CrossRef]

43. Benjamin, Y.; Hochberg, Y. Controlling the false discovery rate: A practical and powerful approach to multiple testing. J. R. Stat. Soc. Ser. B Methodol. 1995, 57, 289-300. [CrossRef] 
44. Kendall, M.G. A new measure of rank correlation. Biometrika 1938, 30, 81-93. [CrossRef]

45. Al-Shaar, L.; Satija, A.; Wang, D.D.; Rimm, E.B.; Smith-Warner, S.A.; Stampfer, M.J.; Hu, F.B.; Willett, W.C. Red meat intake and risk of coronary heart disease among US men: Prospective cohort study. BMJ 2020, 371, m4141. [CrossRef]

46. Jo, G.; Oh, H.; Singh, G.M.; Park, D.; Shin, M.-J. Impact of dietary risk factors on cardiometabolic and cancer mortality burden among Korean adults: Results from nationally representative repeated cross-sectional surveys 1998-2016. Nutr. Res. Pract. 2020, 14, 384-400. [CrossRef]

47. Alba, H.D.; Freitas Júnior, J.E.; Leite, L.C.; Azevêdo, J.A.; Santos, S.A.; Pina, D.S.; Cirne, L.G.A.; Rodrigues, C.S.; Silva, W.P.; Lima, V.G.O; ; et al. Protected or unprotected fat addition for feedlot lambs: Feeding behavior, carcass traits, and meat quality. Animals 2021, 11, 328. [CrossRef]

48. Urrutia, O.; Mendizabal, J.A.; Alfonso, L.; Soret, B.; Insausti, K.; Arana, A. Adipose tissue modification through feeding strategies and their implication on adipogenesis and adipose tissue metabolism in ruminants. Int. J. Mol. Sci. 2020, 21, 3183. [CrossRef] [PubMed]

49. Le, V.H.; Nguyen, D.V.; Nguyen, Q.V.; Malau-Aduli, B.S.; Nichols, P.D.; Malau-Aduli, A.E.O. Fatty acid profiles of muscle, liver, heart and kidney of Australian prime lambs fed different polyunsaturated fatty acids enriched pellets in a feedlot system. Sci. Rep. 2019, 9, 1238. [PubMed]

50. Nguyen, Q.V.; Malau-Aduli, B.S.; Cavalieri, J.; Nichols, P.D.; Malau-Aduli, A.E.O. Enhancing omega-3 long-chain polyunsaturated fatty acid content of dairy-derived foods for human consumption. Nutrients 2019, 11, 743. [CrossRef] [PubMed]

51. Nguyen, D.V.; Le, V.H.; Nguyen, Q.V.; Malau-Aduli, B.S.; Nichols, P.D.; Malau-Aduli, A.E.O. Omega-3 long-chain fatty acids in the heart, kidney, liver and plasma metabolite profiles of Australian prime lambs supplemented with pelleted canola and flaxseed oils. Nutrients 2017, 9, 893. [CrossRef]

52. Celada, P.; Sánchez-Múniz, F.J. Are meat and meat product consumptions harmful? Their relationship with the risk of colorectal cancer and other degenerative diseases. An. Real. Acad. Farm. 2016, 82, 68-90.

53. Gruffat, D.; Durand, D.; Rivaroli, D.; Do Prado, I.; Prache, S. Comparison of muscle fatty acid composition and lipid stability in lambs stall-fed or pasture-fed alfalfa with or without sainfoin pellet supplementation. Animal 2020, 14, 1093-1101. [CrossRef] [PubMed]

54. Renna, M.; Brugiapaglia, A.; Zanardi, E.; Destefanis, G.; Prandini, A.; Moschini, M.; Sigolo, S.; Lussiana, C. Fatty acid profile, meat quality and flavour acceptability of beef from double-muscled Piemontese young bulls fed ground flaxseed. Ital. J. Anim. Sci. 2019, 18, 355-365. [CrossRef]

55. De Lima Júnior, D.M.; do Nascimento Rangel, A.H.; Urbano, S.A.; Moreno, G.M.B. Oxidação lipídica e qualidade da carne ovina. Acta Vet. Bras. 2013, 7, 14-28.

56. Pewan, S.B.; Otto, J.R.; Kinobe, R.T.; Adegboye, O.A.; Malau-Aduli, A.E.O. MARGRA lamb eating quality and human healthpromoting omega-3 long-chain polyunsaturated fatty acid profiles of Tattykeel Australian White sheep: Linebreeding and gender effects. Antioxidants 2020, 9, 1118. [CrossRef] [PubMed]

57. Facciolongo, A.M.; Lestingi, A.; Colonna, M.A.; Nicastro, F.; De Marzo, D.; Toteda, F. Effect of diet lipid source (linseed vs. soybean) and gender on performance, meat quality and intramuscular fatty acid composition in fattening lambs. Small Rum. Res. 2018, 159, 11-17. [CrossRef]

58. Den Hartigh, L.J. Conjugated linoleic acid effects on cancer, obesity, and atherosclerosis: A review of pre-clinical and human trials with current perspectives. Nutrients 2019, 11, 370. [CrossRef] [PubMed]

59. Simopoulos, A.P. Genetic variants in the metabolism of omega- 6 and omega- 3 fatty acids: Their role in the determination of nutritional requirements and chronic disease risk. Exp. Biol. Med. 2010, 235, 785-795. [CrossRef]

60. Urrutia, O.; Mendizabal, J.A.; Insausti, K.; Soret, B.; Purroy, A.; Arana, A. Effects of addition of linseed and marine algae to the diet on adipose tissue development, fatty acid profile, lipogenic gene expression, and meat quality in lambs. PLoS ONE 2016, 11, e0156765. [CrossRef]

61. Widmann, P.; Nuernberg, K.; Kuehn, C.; Weikard, R. Association of an ACSL1 gene variant with polyunsaturated fatty acids in bovine skeletal muscle. BMC Genet. 2011, 12, 1-13. [CrossRef]

62. Lee, J.M.; Lee, H.; Kang, S.; Park, W.J. Fatty acid desaturases, polyunsaturated fatty acid regulation, and biotechnological advances. Nutrients 2016, 8, 23. [CrossRef]

63. Mueller-Harvey, I.; Bee, G.; Dohme-Meier, F.; Hoste, H.; Karonen, M.; Kölliker, R.; Lüscher, A.; Niderkorn, V.; Pellikaan, W.F.; Salminen, J.P.; et al. Benefits of condensed tannins in forages fed to ruminants: Importance of structure, concentration and diet. Crop. Sci. 2019, 59, 861-885. [CrossRef]

64. Vasta, V.; Daghio, M.; Cappucci, A.; Buccioni, A.; Serra, A.; Viti, C.; Mele, M. Invited review: Plant polyphenols and rumen microbiota responsible for fatty acid biohydrogenation, fiber digestion, and methane emission: Experimental evidence and methodological approaches. J. Dairy Sci. 2019, 102, 3781-3804. [CrossRef]

65. Freitas, N.; Araújo, M.; Oliveira, R.; Lanna, D.; Marques, C.; Torreão, J.; Santos, C.B.; Silva Junior, J.M.; Edvan, R.L.; Bezerra, L.R. Production, composition, fatty acid profile and sensory traits of milk from goats fed crude glycerin from waste frying oils used in biodiesel production. Livest. Sci. 2020, 238, 104060. [CrossRef]

66. Wood, J.; Enser, M.; Fisher, A.; Nute, G.; Sheard, P.; Richardson, R.; Hughes, S.I.; Whittington, F.M. Fat deposition, fatty acid composition and meat quality: A review. Meat Sci. 2008, 78, 343-358. [CrossRef] 
67. Lee, S.; Lee, J.; Choi, I.J.; Kim, Y.-W.; Ryu, K.W.; Kim, Y.-I.; Kim, J. Dietary n-3 and n-6 polyunsaturated fatty acids, the FADS gene, and the risk of gastric cancer in a Korean population. Sci. Rep. 2018, 8, 3823. [CrossRef] [PubMed]

68. Calder, P.C. $\mathrm{n}-3$ Polyunsaturated fatty acids, inflammation, and inflammatory diseases. Am. J. Clin. Nutr. 2006, 83, 1505S-1519S. [CrossRef] [PubMed]

69. Yates, C.M.; Calder, P.C.; Rainger, G.E. Pharmacology and therapeutics of omega-3 polyunsaturated fatty acids in chronic inflammatory disease. Pharmacol. Ther. 2014, 141, 272-282. [CrossRef] [PubMed]

70. Yang, B.; Ren, X.-L.; Fu, Y.-Q.; Gao, J.-L.; Li, D. Ratio of n-3/n-6 PUFAs and risk of breast cancer: A meta-analysis of 274135 adult females from 11 independent prospective studies. BMC Cancer 2014, 14, 105. [CrossRef]

71. Lee, J.; Choi, J.; Alpergin, E.S.S.; Zhao, L.; Hartung, T.; Scafidi, S.; Riddle, R.C.; Wolfgang, M.J. Loss of hepatic mitochondrial long-chain fatty acid oxidation confers resistance to diet-induced obesity and glucose intolerance. Cell Rep. 2017, 20, 655-667. [CrossRef] [PubMed]

72. Castillo Vargas, J.A. Metabolism and function of lipids in the adipose and liver tissues of production ruminants: A review. CES Med. Vet. Zootec. 2019, 14, 30-44. [CrossRef]

73. Byelashov, O.A.; Sinclair, A.J.; Kaur, G. Dietary sources, current intakes, and nutritional role of omega-3 docosapentaenoic acid Lipid Technol. 2015, 27, 79-82. [CrossRef]

74. Kuhnt, K.; Degen, C.; Jahreis, G. Evaluation of the impact of ruminant trans fatty acids on human health: Important aspects to consider. Crit. Rev. Food Sci. Nutr. 2016, 56, 1964-1980. [CrossRef]

75. Hennessy, A.A.; Ross, R.P.; Devery, R.; Stanton, C. The health promoting properties of the conjugated isomers of $\alpha$-linolenic acid. Lipids 2011, 46, 105-119. [CrossRef]

76. Gómez, I.; Mendizabal, J.; Sarriés, M.; Insausti, K.; Albertí, P.; Realini, C.; Perez-Juan, M.; Oliver, M.A.; Purroy, A.; Beriain, M.J. Fatty acid composition of young Holstein bulls fed whole linseed and rumen-protected conjugated linoleic acid enriched diets. Livest. Sci. 2015, 180, 106-112. [CrossRef]

77. Guerrero, A.; Sañudo, C.; Campo, M.; Olleta, J.; Muela, E.; Macedo, R.; Macedo, F.A.F. Effect of linseed supplementation level and feeding duration on performance, carcass and meat quality of cull ewes. Small Rum. Res. 2018, 167, 70-77. [CrossRef]

78. Moibi, J.A.; Christopherson, R.J. Effect of environmental temperature and a protected lipid supplement on the fatty acid profile of ovine longissimus dorsi muscle, liver and adipose tissues. Livest. Prod. Sci. 2001, 69, 245-254. [CrossRef]

79. Borowiec, F.; Micek, P.; Marcinski, M.; Barteczko, J.; Zajac, T. Linseed-based diets for sheep. 2. Performance and chemical composition of meat and liver. J. Anim. Feed Sci. 2004, 13, 19-22. [CrossRef]

80. Coleman, D.N.; Martin, A.C.C.; Jin, Y.; Lee, K.; Relling, A.E. Prepartum fatty acid supplementation in sheep. IV. Effect of calcium salts with eicosapentaenoic acid and docosahexaenoic acid in the maternal and finishing diet on lamb liver and adipose tissue during the lamb finishing period. J. Anim. Sci. 2019, 97, 3071-3088. [CrossRef]

81. Bernacka, H.; Peter, E.; Mistrzak, M. Fatty acid content in meat, heart, and liver of conventionally bred Polish Merino lambs. Fatty acid content in meat, heart, and liver of conventionally bred Polish Merino lambs. Med. Weter. 2013, 69, 424-427.

82. Demirel, G.; Wood, J.D.; Enser, M. Conjugated linoleic acid content of the lamb muscle and liver fed different supplements. Small Rum. Res. 2004, 53, 23-28. [CrossRef]

83. Kim, S.C.; Adesogan, A.T.; Badinga, L.; Staples, C.R. Effects of dietary n-6: N-3 fatty acid ratio on feed intake, digestibility, and fatty acid profiles of the ruminal contents, liver, and muscle of growing lambs. J. Anim. Sci. 2007, 85, 706-716. [CrossRef] [PubMed]

84. Biel, W.; Czerniawska-Piątkowska, E.; Kowalczyk, A. Offal chemical composition from veal, beef, and lamb maintained in organic production systems. Animals 2019, 9, 489. [CrossRef] [PubMed]

85. Florek, M.; Litwińczuk, Z.; Skałecki, P.; Kędzierska-Matysek, M.; Grodzicki, T. Chemical composition and inherent properties of offal from calves maintained under two production systems. Meat Sci. 2012, 90, 402-409. [CrossRef] [PubMed]

86. Hicks, T.M.; Knowles, S.O.; Farouk, M.M. Global provisioning of red meat for flexitarian diets. Front. Nutr. 2018, 5, 50. [CrossRef]

87. Mashek, D.G.; Coleman, R.A. Cellular fatty acid uptake: The contribution of metabolism. Curr. Opin. Lipidol. 2006, 17, 274-278. [CrossRef]

88. Hagve, T.A.; Woldseth, B.; Brox, J.; Narce, M.; Poisson, J.P. Membrane fluidity and fatty acid metabolism in kidney cells from rats fed purified eicosapentaenoic acid or purified docosahexaenoic acid. Scand. J. Clin. Lab. Investig. 1998, 58, 187-194. [CrossRef] [PubMed]

89. Liabo, J.; Odden, N.; Christiansen, E.N.; Hagve, T.A. Metabolism of long-chain polyunsaturated fatty acids in rat kidney cells. Ann. Nutr. Metab. 2003, 47, 22-30. [CrossRef] [PubMed]

90. Schaap, F.G.; van der Vusse, G.J.; Glatz, J.F.C. Fatty acid-binding proteins in the heart. Card. Metab. Health Dis. 1998, 180 , 43-51. 